arch-ive/9504012

CSIC-IMAFF-15-1995

April 1995

\title{
Fokker-Planck approach to quantum lattice Hamiltonians
}

\author{
Fernando Jiménez ${ }^{a}$. and Germán Sierra ${ }^{b}$. \\ ${ }^{a}$ Departamento de Aerotecnia, \\ Universidad Politécnica de Madrid. 28040-Madrid, Spain. \\ ${ }^{b}$ Instituto de Matemáticas y Física Fundamental, \\ Consejo Superior Investigaciones Científicas. 28006-Madrid, Spain.
}

\begin{abstract}
Fokker-Planck equations have been applied in the past to field theory topics such as the stochastic quantization and the stabilization of bottomless action theories. In this paper we give another application of the FP-techniques in a way appropriate to the study of the ground state, the excited states and the critical behaviour of quantum lattice Hamiltonians. With this purpose, we start by considering a discrete or lattice version of the standard FPHamiltonian. The well known exponential ansatz for the ground state wave functional becomes in our case an exponential "cluster" expansion. With a convenient choice for this latter, we are able to construct FP-Hamiltonians which to a large extent reproduce critical properties of "realistic" quantum lattice Hamiltonians, as the one of the Ising model in a transverse field (ITF). In one dimension, this statement is made manifest by proving that the FPHamiltonian we built up belongs to the same universality class as the standard ITF model or, equivalently, the 2D-classical Ising model. To this respect, some considerations concerning higher dimensional ITF models are outlined.
\end{abstract}

PACS numbers: 02.90.+p, 05.50.+q, 64.60.Cn, 02.50.Ey

\footnotetext{
${ }^{1}$ E-mail address: IFFJIMENEZ @ROCA.CSIC.ES.

${ }^{2}$ E-mail address: SIERRA @CC.CSIC.ES.
} 


\section{Introduction}

A fundamental problem in Statistical Mechanics and Solid State Physics is the search of the ground state and excitations of many body Hamiltonians. Exact solutions have been obtained for integrable one dimensional Hamiltonians [1], [2], [3]. However, the majority of the models are not integrable, specially in dimensions greater than one, and in these cases one has to resort to approximation techniques. We have squematically,

$$
H(\text { Hamiltonian }) \longrightarrow\left\{\begin{array}{l}
\text { Perturbation theory } \\
\text { Variational methods } \\
\text { Renormalization group } \\
\text { Numerical approaches } \\
\vdots
\end{array}\right\} \longrightarrow\left|\psi_{0}\right\rangle \text { (ground state) }
$$

The path from $H$ to $\left|\psi_{0}\right\rangle$ is very cumbersome and can only be fully accomplished in a few cases. Instead, one could try to reverse the arrows in (1.1), by making a clever choice of the state $\left|\psi_{0}\right\rangle$ first, and asking latter for the Hamiltonian $H$ for which the proposed ansatz becomes the exact ground state (this second strategy can be paraphrased in the words of [4] as "finding questions to some interesting answers". Some remarkable cases where this "inverse method" has been applied would include the exponential Jastrow wave functions, used in the study of liquid ${ }^{4} \mathrm{He}$ [5] and nuclear matter [6], the valence-bond solid states (VBS) [7], the Laughlin wave function [8], etc. The path from $\left|\psi_{0}\right\rangle$ to $H$ could be summarized as follows:

$$
\left|\psi_{0}\right\rangle \longrightarrow\left\{\begin{array}{l}
R_{\alpha}\left|\psi_{0}\right\rangle=0 \\
\alpha=1, \cdots, N
\end{array}\right\} \longrightarrow\left\{\begin{array}{l}
H=\sum_{\alpha=1}^{N} R_{\alpha}^{\dagger} R_{\alpha} \\
H\left|\psi_{0}\right\rangle=0
\end{array}\right\}
$$

where $\alpha$ denotes either sites in a lattice, or particles or some other quantum numbers. In words, one has first to find a set of operators $R_{\alpha}$ which annihilate the state $\left|\psi_{0}\right\rangle$; afterwards one builts up a positive semidefinite Hamiltonian $H$ according to the prescription (1.2). If the state $\left|\psi_{0}\right\rangle$ of (1.2) is a valence-bond state, then the $R_{\alpha}$ are projector operators (i.e. $R_{\alpha}^{\dagger}=R_{\alpha}, R_{\alpha}^{2}=R_{\alpha}$ ) and we can write $H=\sum_{\alpha} R_{\alpha}$. However, for Jastrow type wave functions, the $R_{\alpha}$ are not projectors, and these will be the kind of operators which we shall be concerned with.

The aim of this paper is to combine the direct and inverse methods (1.1)-(1.2) in the study of quantum lattice Hamiltonians. To be more concrete, the problem we want to adress is the construction of the ground state $\left|\psi_{0}\right\rangle$ and the excited states of a quantum Hamiltonian $H$ defined on a lattice. The strategy could be summarized in the following diagram:

$$
\begin{aligned}
& \text { Perturbation } \quad \text { Fokker - Planck } \\
& H \stackrel{\text { theory }}{\longrightarrow} \psi(\text { Exponential ansatz }) \stackrel{\text { method }}{\longrightarrow} \quad H^{F P}
\end{aligned}
$$

The first step consists in the construction of a state of exponential type, which will be our candidate for the ground state of $H$. To make this ansatz, we shall 
follow the perturbative-variational procedure of determination of the ground state $\left|\psi_{0}(\lambda)\right\rangle$ of a lattice Hamiltonian, in the form $H(\lambda)=H_{0}+\lambda H_{1}$, established in [9]; here $H_{0}$ is a Hamiltonian whose non degenerated ground state $|0\rangle$ is known, and $\lambda$ is a coupling constant.

Two alternative ways for writting the state $\left|\psi_{0}(\lambda)\right\rangle$ of $H(\lambda)$ were proposed in reference [9], to wit:

$$
\left|\psi_{0}(\lambda)\right\rangle=\exp \left(\sum_{n \geq 1} \lambda^{n} U_{n}\right)|0\rangle
$$

or

$$
\left|\psi_{0}(\lambda)\right\rangle=\exp \left(\sum_{I} \alpha_{I}(\lambda) V_{I}\right)|0\rangle
$$

where both $U_{n}$ and $V_{I}$ are a certain set of interrelated local operators (see below), whereas the $\alpha_{I}$ are certain functions of $\lambda$.

Expansion (1.4) is adequate to make contact with the standard perturbation theory (PT), whereas expansion (1.5) is a kind of cluster expansion. Let us describe in some more detail the main ideas of [9], since this paper is, after all, an extension of that work.

When one expands the exponential of (1.4) and compares the result with the usual PT, one obtains a set of equations for the operators $U_{n}$. Thus, we can say that PT to order $\nu$ fixes, up to some freedom, the familly of operators $\left\{U_{n}\right\}_{1 \leq n \leq \nu}$. Working through particular examples, as the Ising model in a transverse field, one observes that each operator $U_{n}$ can in fact be written as a sum of "irreducible" operators $V_{I}$ :

$$
U_{n}=\sum_{I} p_{n, I} V_{I}
$$

where in [9] the label $I$ denoted a cluster formed by a finite number of 1 's distributed on certain sites of the lattice, the rest of sites being occupied by zeros. The operator $V_{I}$ obeys a fusion algebra given by $V_{I} V_{J}=V_{I+J}$, in such a way that the cluster $I+J$ is obtained through the usual $\mathbf{Z}_{2}$-sum rules: $0+0=1+1=0,0+1=1+0=$ 1 (this rule has its origin in the $\mathbf{Z}_{2}$-symmetry of the Ising model). The usefulness of eq.(1.6) lies in the fact that a given cluster operator $V_{I}$ may appear at different -and possible at all- orders in PT. This would imply that for a fixed value of $I, p_{n, I}$ might have an infinite number of non zero values. Introducing (1.6) in (1.4) we can relate the perturbative and cluster expansions with:

$$
\alpha_{I}(\lambda)=\sum_{n \geq \nu_{I}} p_{n, I} \lambda^{n}
$$

where the number

$$
\nu_{I}=\operatorname{minimun}\left\{n / p_{n, I} \neq 0\right\}
$$

is called the level of the operator $V_{I}$; somehow $\nu_{I}$ measures the size of the cluster [9]. Definition (1.7) implies that $\alpha_{I}(\lambda)$ has a Taylor expansion starting at $\lambda^{\nu_{I}}$ and containing non vanishing terms of higher powers whenever $V_{I}$ appears in $U_{n}$. 
If we think of $p_{n, I}$ as a matrix with two entries $n$ and $I$-or rather $\nu_{I^{-}}$, we see by its definition that $p_{n, I}$ is a lower triangular matrix of infinite dimension (see fig.1a). Thus PT to order $\nu$ gives all the entries of $p_{n, I}$ to a "depth" $\nu$, i.e $\left\{p_{n, I}\right\}_{1 \leq n \leq \nu}$ (see fig.1b).

However, eq.(1.5) provides another approach to the exact ground state. Indeed, the knowledge of a single function $\alpha_{I}(\lambda)$ for a given operator $V_{I}$ involves all orders in PT. This result suggests a non-perturbative approach to $\left|\psi_{0}\right\rangle$ based on the truncation of (1.5) to just those operators $V_{I}$ with an order less or equal to a given order $\nu$ (see fig.1c):

$$
\left|\psi^{(\nu)}\right\rangle=\exp \left(\sum_{I, \nu_{I} \leq \nu} \alpha_{I}^{(\nu)} V_{I}\right)|0\rangle
$$

In [9] the functions $\alpha_{I}^{(\nu)}$ were fixed by using the state $\left|\psi^{(\nu)}\right\rangle$ as a trial variational state for the Hamiltonian $H(\lambda)=H_{0}+\lambda H_{1}$. In this case the variational ansatz agrees to order $\nu$ with the perturbative ground state and gives non perturbative predictions for large values of $\lambda$, where PT cannot be trusted.

In this paper we propose an alternative way for computing the parameters $\alpha_{I}^{(\nu)}$. The principle of our method is a generalization of the well known Fokker-Planck's procedure, by means of which one associates a Hamiltonian to a wave function having an exponential form (see appendix A, for an application to Quantum Mechanics). The rule is simply to act with $H_{0}$ on the proposal (1.9) until one arrives to a FokkerPlanck Hamiltonian $H^{F P,(\nu)}\left(\alpha_{I}^{(\nu)}\right)$ which depends, of course, on the whole set of parameters $\alpha_{I}^{(\nu)}$ entering in the definition of $\left|\psi^{(\nu)}\right\rangle$. The condition we use to fix these parameters as functions of $\lambda$ is that $H^{F P,(\nu)}$ should only differ from $H$ in operators of a level greater than $\nu$, i.e:

$$
H-H^{F P,(\nu)}=\sum_{I, \nu_{I} \geq \nu} C_{I}^{(\nu)}(\lambda) V_{I}
$$

Correspondingly, the FP-energy $E^{F P,(\nu)}(\lambda)$ although incorporating non perturbative effects, should agree with the exact ground state energy up to order $\lambda^{\nu+1}$. Compairing $E^{F P, \nu}(\lambda)$ (for $\nu=1$ and 2) with the variational energy $E^{v a r, \nu}(\lambda)$ obtained in [9] in the study of the Ising model in a transverse field, one observes that the latter gives better values than the former, as to the energy is concerned. It seems natural then to wonder about the advantages of the FP-approach versus the variational one. Well, the problem is that within the framework of the variational approach is very difficult to go to higher values of $\nu$ since, for example, the norm of the variational state (1.9) turns out to be the partition function of a statistical mechanical model whose couplings are dictated by the operators $V_{I}$. On the contrary, in the FP procedure the norm of the state (1.9) is not needed to fix the parameters $\alpha_{I}^{(\nu)}$ in terms of $\lambda$; rather, what is required is the algebra satisfied by the cluster operators $V_{I}$, and this algebra is relatively simple in the example considered in this paper. Besides, within the FP-method we can always match the perturbative results to any order, at the expense of including a sufficient number of operators in the ansatz (1.9). It is clear, however, that except for the lowest orders in PT the complexity of the ansatz will obscure its usefulness. On the other hand, one of the aims in constructing more general ansatzs for ground states is to see whether or not 
they exhibit critical behaviour. In this sense, what actually matters is to determine the universality class which this Hamiltonians belongs to, rather than their apparent simplicity. Bearing in mind these ideas, we can try to reinterpret eq.(1.3) as a map from a given Hamiltonian $H$ to a FP-Hamiltonian $H^{F P}$ belonging to the same universality class as $H$; in this case $H$ and $H^{F P}$ would share the same critical properties. To achieve this map one should consider ansatzs involving cluster operators $V_{I}$ with all the possible sizes (see fig.1d). We shall show in this paper an example where such a construction can be accomplished.

The organization of the paper is as follows. In section 2 we review the application of the FP machinery to field theory in the continuum and reconsider it in such a way that it can be applied to lattice Hamiltonians. In section 3 we benefit from this generalization to look into the Ising model in a transverse field (ITF) in $D=$ 1 and $D=2$ dimensions. In section 4 we generalize the exponential ansatz of section 3 in order to include operators at all length scales, and we show in the one dimensional case the existence of a critical behaviour for a particular value of the coupling constant. In section 5 we study the problem of the excited states of FP-Hamiltonians using a "change of variables" which brings the Hamiltonian to a simpler form $H^{R F P}$, which we call "reduced" FP-Hamiltonian. Finally, a general discussion with prospects for the future is proposed in section 6 .

We have also included four appendices which illustrate in detail some aspects mentioned in the course of the paper. In particular we collect in appendix D the results of sections 3 and 4 extended to the case of ITF models in dimensions higher than one.

\section{Fokker-Planck lattice hamiltonians: General construction}

The Fokker Planck Hamiltonians arise naturally in the study of stochastics processes in Statistical Mechanics or Quantum Field Theories. The idea is to consider a particle or a field evolving in time under the combined action of a force and a gaussian noise [10]. The evolution equation for such a field $\phi(x, t)$ is given by the Langevin equation:

$$
\frac{\partial \phi(x, t)}{\partial t}=-\frac{\delta S[\phi]}{\delta \phi}+\eta(x, t)
$$

where $S[\phi]$ is the potential and $\eta(x, t)$ is the noise. Here $x \in \mathbf{R}^{D}$ is the space location of the field. The corresponding probability $P[\phi, t]$ for finding a given configuration $\phi(x)$ at the time $t$ is governed by the Fokker-Planck (FP) equation. A suggestive way of writing this equation comes up if we make the change of variables $P[\phi, t]=$

$e^{-\frac{S[\phi]}{2}} \psi[\phi, t]$; with this change, the FP equation for $\psi[\phi, t]$ becomes a Schrödinger like equation in imaginary time, namely:

$$
\frac{\partial \psi[\phi, t]}{\partial t}=-H^{F P} \psi[\phi, t]
$$

where 


$$
H^{F P}=\frac{1}{2} \int d^{D} x\left[-\frac{\delta^{2}}{\delta \phi^{2}}+\frac{1}{4}\left(\frac{\delta S}{\delta \phi}\right)^{2}-\frac{1}{2} \frac{\delta^{2} S}{\delta \phi^{2}}\right]
$$

is called the Fokker-Planck Hamiltonian. To this point, several comments are in order:

(i) $H^{F P}$ is a positive semidefinite operator. This follows inmmediately if we write the Hamiltonian $H^{F P}$ as :

$$
H^{F P}=\frac{1}{2} \int d^{D} x R^{\dagger}(x) R(x)
$$

where

$$
R(x)=i \frac{\delta}{\delta \phi(x)}+\frac{i}{2} \frac{\delta S}{\delta \phi(x)}
$$

(ii) The functional $\psi_{0}[\phi]=e^{-\frac{S[\phi]}{2}}$ is annihilated by the family of commuting operators $R(x)$ (i.e. $R(x) R(y)=R(y) R(x)$ )

$$
R(x) \psi_{0}=0, \quad \forall x
$$

Hence, if $\psi_{0}$ is a normalized state, then it becomes a ground state of the FP-Hamiltonian (2.3).

(iii) The norm of the ground state $\psi_{0}[\phi]$ can be interpreted as the partition function of a statistical mechanical model or euclidean quantum field theory in $D$ dimensions, with Boltzmann weights $e^{-S[\phi]}$

$$
Z \equiv\left\langle\psi_{0} \mid \psi_{0}\right\rangle=\int D \phi e^{-S[\phi]}
$$

These simple properties have been used in a variety of applications, some of which are worth mentioning. To begin with, let us quote the stochastic quantization method of Parisi and $\mathrm{Wu}$ [11. Introducing a ficticious time (the fith-time), this procedure enables one to compute quantum mechanical expectation values of observables $Q[\phi]$ in terms of time averages of fields evolving under the Langevin equation (2.1):

$$
\langle Q\rangle=\frac{1}{Z} \int D \phi Q[\phi] e^{-S[\phi]}=\lim _{T \rightarrow \infty} \frac{1}{T} \int_{0}^{T} Q[\phi(x, t)] d t
$$

This formulation has been applied to quantum field theories, including gauge theories.

Another fruitful application carried out in reference [12] concerns to the stabilization of bottomless field theories, i.e. theories with a potential unbounded from below and, therefore, with divergent partition functions. Here one uses the unbounded action $S[\phi]$ and eq.(2.3) to construct a FP-Hamiltonian, which thanks to property (i) is well defined. Since $H^{F P}$ is bounded from below, the corresponding 
ground state $\psi_{0}^{\text {nor }}$ will be normalizable; that gives rise to a bounded effective action $S_{\text {eff }}[\phi]$ through the definition $\psi_{0}^{n o r} \equiv e^{-\frac{S_{e f f}[\phi]}{2}}$. This stabilization procedure has also been applied to a number of theories including gravity [13], and 2D-matrix models [14, [15]

Finally, we want to mention the connection between FP-Hamiltonians and supersymmetry. Let us suppose that we enlarge the field theory presented earlier with a pair of anticommuting fields $\theta(x)$ and $\bar{\theta}(y)$ satisfying the anticommutation relations:

$$
\begin{gathered}
\{\theta(x), \theta(y)\}=\{\bar{\theta}(x), \bar{\theta}(y)\}=0 \\
\{\theta(x), \bar{\theta}(y)\}=\delta(x-y)
\end{gathered}
$$

Now we define two supersymmetric charges $Q$ and $\bar{Q}$

$$
\begin{gathered}
Q=\int d x R(x) \theta(x) \\
\bar{Q} \equiv Q^{\dagger}=\int d x R^{\dagger}(x) \bar{\theta}(x)
\end{gathered}
$$

and satisfying

$$
Q^{2}=\bar{Q}^{2}=0
$$

Then it is easy to see that the eq.(2.6) characterizing the state $\psi_{0}$ can be translated into the equations

$$
Q \psi_{0}=\bar{Q} \psi_{0}=0
$$

In terms of $Q$ and $\bar{Q}$ one can define a supersymmetric Hamiltonian $H_{S S}$ whose restriction to the bosonic sector (i.e $\bar{\theta}=0$ ) is nothing else but the FP-Hamiltonian (2.3), namely:

$$
\begin{gathered}
H_{S S}=\frac{1}{2}(Q \bar{Q}+\bar{Q} Q) \\
=H^{F P}+\frac{1}{2} \int d^{D} x \frac{\delta^{2} S}{\delta \phi(x)^{2}}[\theta(x), \bar{\theta}(x)]
\end{gathered}
$$

In the SUSY-terminology we can say that the exponential state $\psi_{0}=e^{-\frac{S[\phi]}{2}}$ is a supersymmetric ground state [16].

The connection between the FP-method and SUSY is very suggestive but we shall not pursued it here, except for a discrete version of eqs.(2.9)-(2.15).

As for the use of FP-ideas we make in this paper, let us say that they can be simply illustrated in the continuum as follows. Suppose we are given a field theoretical hamiltonian:

$$
H=\int d^{D} x\left[-\frac{1}{2} \frac{\delta^{2}}{\delta \phi^{2}}+U(\phi)\right]
$$


where $U(\phi)$ is a funtion of $\phi(x), \nabla \phi(x)$, etc. Then if we look for the ground state of $H$ in the form $\psi_{0}[\phi]=e^{-\frac{S[\phi]}{2}}$, it turns out that the potential $U(\phi)$ and the ground state energy $E_{0}$ are related to $S(\phi)$ by the eq.

$$
U(\phi)-E_{0}=\frac{1}{8}\left(\frac{\delta S}{\delta \phi}\right)^{2}-\frac{1}{4} \frac{\delta^{2} S}{\delta \phi^{2}}
$$

Eq. (2.17) is a functional version of the Ricatti equation [17]. Consequently, the eigenvalue problem for the ground state of $H$ is reduced, assuming the exponential ansatz $\psi_{0}[\phi]=e^{-\frac{S[\phi]}{2}}$, to the solution of the functional Ricatti equation (2.17).

In the context of Quantum Mechanics the analogue of eq.(2.17) can be taken as the starting point of a perturbative-variational method [17] very close in spirit to the one developed in [9]. There are situations where the field theoretical Hamiltonian (2.16) can be diagonalized exactly, for example in terms of creation and annihilation operators as in pure QED [18]. The corresponding wave functional $\psi_{0}[\phi]$ is then essentially a gaussian. When interactions are present, matters are not so simple. In any case, before looking for the ground state of quantum field Hamiltonians defined in the continuum, one should first regularize the model so as to control the infinities and this can be done, for example, by using a lattice regularization. This is, therefore, another motivation to consider the FP-method in the context of quantum lattice Hamiltonians.

In the remaining of this section we shall present a generalization of the FP techniques introduced above. From an abstract point of view, the basic elements required in a FP-construction are: a) an algebra of operators, b) a vacuum state $|0\rangle$, c) a free Hamiltonian $H_{0}$ and d) an action $S$. In the continuum FP-construction, these elements are given respectively by the operators $\frac{\delta}{\delta \phi(x)}$ and $\phi(x)$, the free Hamiltonian $H_{0}=-\frac{1}{2} \int d^{D} x \frac{\delta^{2}}{(\delta \phi(x))^{2}}$ whose ground state is the constant wave function, and the action $S[\phi]$ which is a functional of the field $\phi(x)$.

The construction can be implemented as follows:

a) Algebra of operators. We consider a family of unitary operators $\left\{X_{a}\right\}_{a \in \mathcal{C}_{0}}$, $\left\{Z_{A}\right\}_{A \in \mathcal{C}_{1}}$ satisfying the relations:

$$
\begin{gathered}
X_{a} X_{b}=X_{b} X_{a} \\
Z_{A} Z_{B}=Z_{B} Z_{A} \\
X_{a} Z_{A}=\omega_{a A} Z_{A} X_{a}
\end{gathered}
$$

where the $\omega_{a A}$ are phases $\left(\left|\omega_{a A}\right|=1\right)$. The labels $a$ and $A$ run, respectively, over discrete sets $\mathcal{C}_{0}$ and $\mathcal{C}_{1}$, whose nature will be specific for each model. In practical examples they will denote sites, links, plaquettes or internal quantum numbers.

We shall demand that if $\mathcal{C}_{0}$ and $\mathcal{C}_{1}$ contain an operator, say $X_{a}$ or $Z_{A}$, then they must also contain their hermitean conjugated, i.e:

$$
X_{a}^{\dagger}=X_{\bar{a}} \quad, \quad a, \bar{a} \in \mathcal{C}_{0}
$$




$$
Z_{A}^{\dagger}=Z_{\bar{A}} \quad, \quad A, \bar{A} \in \mathcal{C}_{1}
$$

The Hilbert space where the operators $X_{a}$ and $Z_{A}$ are acting will be denoted by $\mathcal{H}$. The algebra (2.18) is actually the algebra satisfied by the order-disorder variables of, for example, the Ising model, the Potts model or the $\mathbf{Z}_{N}$ gauge models.

b) Vacuum vector. We shall suppose that in the Hilbert space $\mathcal{H}$ there is a vacuum vector $|0\rangle$ satisfying:

$$
X_{a}|0\rangle=|0\rangle \quad, \quad \forall a \in \mathcal{C}_{0}
$$

The existence of a common eigenvector for all the $X_{a}$-operators is in principle possible, because all these operators commute among themselves. Nevertheless, this vector needs not to be unique, i.e. there may exist other vectors satisfying (2.20); this will be the case of models with a broken phase.

c) Free Hamiltonian $H_{0}$. Let us define the following positive semidefinite Hamiltonian:

$$
H_{0}=\frac{1}{2} \sum_{a \in \mathcal{C}_{0}}\left(X_{a}^{\dagger}-\mathbf{1}\right)\left(X_{a}-\mathbf{1}\right)
$$

which, using the unitarity properties of the $X_{a}$ operators and eq.(2.19) transforms in

$$
H_{0}=\sum_{a \in \mathcal{C}_{0}}\left(1-X_{a}\right)
$$

With this definition, it is clear that the vacuum state $|0\rangle$ is a ground state of $H_{0}$

$$
H_{0}|0\rangle=0
$$

d) Exponential ansatz. Suppose we are given a generic operator $S\left(Z_{1}, Z_{2}, \cdots\right)=$ $S\left[Z_{A}\right]$, which we shall call the action operator, and constructed out of the family of commuting $Z$-operators. From $S\left[Z_{A}\right]$ we introduce the state:

$$
\left|\psi_{0}\right\rangle=e^{-\frac{1}{2} S\left[Z_{A}\right]}|0\rangle
$$

It is easy to check that the action of an operator $X_{a}$ on $|0\rangle$ is given by:

$$
\begin{gathered}
X_{a}\left|\psi_{0}\right\rangle=e^{-\frac{1}{2} S\left[\omega_{a A} Z_{A}\right]} X_{a}|0\rangle \\
=e^{-\frac{1}{2} S\left[\omega_{a A} Z_{A}\right]} e^{\frac{1}{2} S\left[Z_{A}\right]}\left|\psi_{0}\right\rangle \equiv e^{\Delta_{a}(S)}\left|\psi_{0}\right\rangle
\end{gathered}
$$

where we have defined

$$
\Delta_{a}(S)=\frac{1}{2}\left(S\left[Z_{A}\right]-S\left[\omega_{a A} Z_{A}\right]\right)
$$

For latter purposes we shall write eq. (2.25) as 


$$
\begin{gathered}
R_{a}\left|\psi_{0}\right\rangle=0 \\
R_{a} \equiv e^{-\Delta_{a}(S)} X_{a}-\mathbf{1}
\end{gathered}
$$

Incidentally, it is noteworthy the commutation relation for the $R$ operators

$$
\left[R_{a}, R_{b}\right]=0
$$

At this moment we may wonder whether there is a Hamiltonian for which the state $\left|\psi_{0}\right\rangle$ becomes an exact ground state. There are several answers to this question but in this paper we propose the following:

Fokker-Planck Hamiltonian. Let $\Omega_{a}$ be a hermitian $\left(\Omega_{a}^{\dagger}=\Omega_{a}\right)$ and positive semidefinite operator $\left(\Omega_{a} \geq 0\right)$; then we shall call a FP-Hamiltonian the following hermitian positive semidefinite operator:

$$
H^{F P}=\frac{1}{2} \sum_{a \in \mathcal{C}_{0}} R_{a}^{\dagger} \Omega_{a} R_{a}
$$

Obviously the state (2.24) is a ground state for this Hamiltonian

$$
H^{F P}\left|\psi_{0}\right\rangle=0
$$

Unfortunately, the choice $\Omega_{a}=1$ although natural, gives rise to Hamiltonians where the operators $X_{a}$ and $Z_{A}$ are mixed together. Yet, a peculiarity of this choice is that the corresponding FP-Hamiltonian presents a hidden supersymmetry. Following the same steps as in the continuum, this suppersymmetry can be made manifest if we introduce a discrete set of anticommuting operators $\theta_{a}$ and $\bar{\theta}_{b}$ satisfying:

$$
\begin{gathered}
\left\{\theta_{a}, \theta_{b}\right\}=\left\{\bar{\theta}_{a}, \bar{\theta}_{b}\right\}=0 \\
\left\{\theta_{a}, \bar{\theta}_{b}\right\}=\delta_{a b}
\end{gathered}
$$

The supersymmetric generators analogues to eqs.(2.11)-(2.12) read now

$$
\begin{aligned}
& Q=\sum_{a \in \mathcal{C}_{0}} R_{a} \theta_{a} \\
& \bar{Q}=\sum_{a \in \mathcal{C}_{0}} R_{a}^{\dagger} \bar{\theta}_{a}
\end{aligned}
$$

and verify $Q^{2}=\bar{Q}^{2}=0$.

The SUSY FP-Hamiltonian is then

$$
\begin{gathered}
H_{S S}^{F P}=\frac{1}{2}(Q \bar{Q}+\bar{Q} Q) \\
=\frac{1}{2} \sum_{a \in \mathcal{C}_{0}} R_{a}^{\dagger} R_{A}+\frac{1}{2} \sum_{a \in \mathcal{C}_{0}}\left[R_{a}, R_{a}^{\dagger}\right] \theta_{a} \bar{\theta}_{a}
\end{gathered}
$$


Hence in the bosonic sector $H_{S S}^{F P}$ reduces to eq.(2.29) with $\Omega_{a}=1$. The problem with the Hamiltonian (2.35) is that it mixes the $X$ and $Z$ operators in a complicated form, namely:

$$
H_{\Omega_{a}=1}^{F P}=\sum_{a \in \mathcal{C}_{0}} \cosh \left(\Delta_{a}(S)\right)\left(e^{\Delta_{a}(S)}-X_{a}\right)
$$

For this reason, and in spite of its intrinsic theoretical interest, we shall not pursue the study of (2.35) in this paper.

From now on, we shall consider the choice $\Omega_{a}=e^{\Delta_{a}(S)}$, which is an hermitian and positive semidefinite operator whenever $S$ is hermitian. In this case, $H^{F P}$ takes a simple form

$$
\left\{\begin{array}{l}
\Omega_{a}=e^{\Delta_{a}(S)} \\
S^{\dagger}=S
\end{array}\right\} \quad \longrightarrow \quad H^{F P}=\sum_{a \in \mathcal{C}_{0}}\left(e^{\Delta_{a}(S)}-X_{a}\right)
$$

or in words, $H^{F P}$ is the sum of the free Hamiltonian (2.22) and an interacting Hamiltonian which depends only on the $Z$-operators.

The previous results are the starting point of our method. Thus, let us suppose we are given a Hamiltonian of the type

$$
H=-\sum_{a \in \mathcal{C}_{0}} X_{a}+U\left[Z_{A}\right]
$$

and whose ground state $\psi_{0}$ we would like to determine in the usual way:

$$
H \psi_{0}=E_{0} \psi_{0}
$$

A solution of this problem arises if one is able to find an action operator $S\left[Z_{A}\right]$ which satisfies the Ricatti equation

$$
U\left[Z_{A}\right]-E_{0}=\sum_{a \in \mathcal{C}_{0}} e^{\Delta_{a}(S)}
$$

For the eigenvalue problem (2.39) the operator $U$ as defined in eq.(2.40) is a datum whereas $S$ is an unknown; however, as it happens in the continuum, one can reverse the roles and take eq.(2.40) as defining the interaction term which corresponds to a given state of the form (2.24). In the sequel, we shall use both kinds of interpretations.

Finally, to complete our generalization of the FP-method we shall study the norm of the exponential state (2.24). If $S$ is an hermitian operator, the norm of this state can be considered as the partition function of an statistical mechanical model with real and positive Bolztmann weights. To show this, we can select an orthonormal basis $\{|I\rangle\}$ of the Hilbert space $\mathcal{H}$ where the $Z$-operators are diagonal

$$
Z_{A}|I\rangle=z_{A, I}|I\rangle
$$

Then the norm of the state (2.24) becomes:

$$
Z=\left\langle\psi_{0} \mid \psi_{0}\right\rangle=\left\langle 0\left|e^{-S\left[Z_{A}\right]}\right| 0\right\rangle=\sum_{I}|\langle 0 \mid I\rangle|^{2} e^{-S\left[z_{A, I}\right]}
$$


In most of the examples $\langle 0 \mid I\rangle$ will be a constant and that implies that the Boltzmann weights are given by $e^{-S\left[z_{A, I}\right]}$.

Before we apply this construction to particular examples, let us mention that a realization of the algebra (2.18) in the continuum can be developed in terms of the Weyl operators:

$$
\begin{gathered}
X_{\vec{r}}=e^{i \hbar \Pi(\vec{r})}=e^{\hbar \frac{\delta}{\delta \phi(\vec{r})}} \\
Z_{\vec{r}}=e^{\phi(\vec{r})}
\end{gathered}
$$

These operators satisfy the algebra

$$
X_{\vec{r}_{1}} Z_{\vec{r}_{2}}=e^{i \hbar \delta\left(\vec{r}_{1}-\vec{r}_{2}\right)} Z_{\vec{r}_{2}} X_{\vec{r}_{1}}
$$

but obviously this expression should be regularized in order to build a consistent theory.

The FP Hamiltonian given by eq. (2.3) can be obtained just by taking the limit $\hbar \rightarrow 0$ of the corresponding (2.37) (see Appendix A). Thus our generalization accounts to a "deformation" of the usual FP-approaches. The word deformation is used here with a meaning very similar to the one used in the theory of quantum groups [19], [20].

\section{The Ising model in a transverse field}

As a prior example of the usefulness of the FP-method outlined in the previous section, let us consider the case of the $D$-dimensional Ising model in a transverse field (ITF).

The model is defined on a hypercubic lattice $\mathbf{Z}_{L}^{D}$ with $N=L^{D}$ sites and Hamiltonian [21, 22, 23]:

$$
\begin{aligned}
& H_{\text {Ising }, D}=H_{0}+U_{\text {Ising }, D} \\
& =-\sum_{\mathbf{j}} \sigma_{\mathbf{j}}^{x}-\lambda \sum_{\mathbf{j}, \mu} \sigma_{\mathbf{j}}^{z} \sigma_{\mathbf{j}+\mu}^{z}
\end{aligned}
$$

where $\sigma_{\mathbf{j}}^{x}, \sigma_{\mathbf{j}}^{z}$ are Pauli matrices acting at the site $\mathbf{j}$, with $\mathbf{j}=\left(j_{1}, \cdots, j_{D}\right), j_{a}=$ $1, \cdots, L$ and $\mu$ is any of the lattice vectors $\mu_{1}=(1,0, \cdots, 0), \cdots, \mu_{D}=(0,0, \cdots, 1)$. These matrices give a representation of the algebra (2.18) for $k=2$. We shall assume in (3.1) periodic boundary conditions, i.e $\sigma_{\mathbf{j}+L \mu_{i}}^{x, z}=\sigma_{\mathbf{j}}^{x, z}, \quad \forall i$.

It is well known that the ground state of (3.1) for $\lambda$ smaller than a certain $D$ depending critical value $\lambda_{c}$ is non degenerate. This accounts to a disordered regime where the order parameter $\left\langle\sigma_{\mathbf{j}}^{z}\right\rangle$ vanishes. For $\lambda>\lambda_{c}$, however, the $\mathbf{Z}_{2}$-symmetry is broken spontaneously and there are two ground states in the termodynamic limit with $\left\langle\sigma_{\mathbf{j}}^{z}\right\rangle \neq 0$. This is the ordered regime of the ITF model.

Let us focus our discussion on the disordered region, where the ground state is non degenerate. Our aim is, for the moment, to construct this ground state.

It is easy to check that all the ingredients needed to apply the generalized FPconstruction are fulfilled by the ITF model. Indeed the algebra of operators $X$ and 
$Z$ are given by the Pauli matrices $\left\{\sigma_{j}^{x}\right\}$ and $\left\{\sigma_{j}^{z}\right\}$, respectively. The vacuum vector $|0\rangle$ is defined by:

$$
|0\rangle=\prod_{j=1}^{N}|0\rangle_{j}
$$

where

$$
\sigma_{j}^{x}|0\rangle_{j}=|0\rangle_{j}, \forall j=1, \cdots, N
$$

The free Hamiltonian (2.22) is, apart from a constant, the same as the free Hamiltonian in eq.(3.1). The problem is therefore to look for the ground state of (3.1) in terms of the exponential ansatz

$$
\left|\psi_{0}\right\rangle=e^{-\frac{1}{2} S\left(\sigma_{1}^{z}, \cdots, \sigma_{N}^{z}\right)}|0\rangle
$$

The corresponding Ricatti equation which fixes $S$ becomes in this context:

$$
\lambda \sum_{\mathbf{j}, \mu} \sigma_{\mathbf{j}}^{z} \sigma_{\mathbf{j}, \mu}^{z}+E_{0}=-\sum_{j=1}^{N} e^{\Delta_{j}(S)}
$$

where

$$
\Delta_{j}(S)=\frac{1}{2}\left[S\left(\sigma_{1}^{z}, \cdots, \sigma_{j}^{z}, \cdots, \sigma_{N}^{z}\right)-S\left(\sigma_{1}^{z}, \cdots,-\sigma_{j}^{z}, \cdots, \sigma_{N}^{z}\right)\right]
$$

The solution of this equation is rather cumbersome, even though in principle it can be obtained successively, step by step, as follows. To start with, suppose we choose for the functional $S$ in (3.4) the expression:

$$
S^{(1)}=-\alpha_{1} \sum_{\mathbf{j}, \mu} \sigma_{\mathbf{j}}^{z} \sigma_{\mathbf{j}+\mu}^{z}
$$

To this choice, it corresponds a state $\psi^{(1)}=e^{-\frac{1}{2} S^{(1)}}|0\rangle$ which can be thought of as the ground state of a $D$-dimensional FP-Hamiltonian:

$$
\begin{gathered}
H_{\text {Ising }, D}^{F P,(1)} \equiv H_{0}+U_{\text {Ising }, D}^{F P,(1)}-E_{\text {Ising }, D}^{F P,(1)} \\
=-\sum_{j=1}^{N}\left(\sigma_{j}^{x}-e^{\Delta_{j}\left(S^{(1)}\right)}\right)=\frac{1}{2} \sum_{j=1}^{N} R_{j}^{(1) \dagger} e^{\Delta_{j}\left(S^{(1)}\right)} R_{j}^{(1)}
\end{gathered}
$$

where

$$
R_{j}^{(1)}=\left(e^{-\Delta_{j}\left(S^{(1)}\right)} \sigma_{j}^{x}-1\right)
$$

and whose interaction potential $U_{I s i n g, D}^{F P,(1)}$ and energy $E_{I s i n g, D}^{F P,(1)}$ are given by:

$$
\begin{gathered}
U_{\text {Ising }, D}^{F P,(1)}-E_{I s i n g, D}^{F P,(1)}=\sum_{j=1}^{N} e^{\Delta_{j}\left(S^{(1)}\right)}=\sum_{\mathbf{j}} e^{-\alpha_{1} \sum_{\mu} \sigma_{\mathbf{j}}^{z}\left(\sigma_{\mathbf{j}+\mu}^{z}+\sigma_{\mathbf{j}-\mu}^{z}\right)} \\
=\left(\cosh \alpha_{1}\right)^{2 D} \sum_{\mathbf{j}} \prod_{\mu}\left(1-t_{1} \sigma_{\mathbf{j}}^{z} \sigma_{\mathbf{j}+\mu}^{z}\right)\left(1-t_{1} \sigma_{\mathbf{j}}^{z} \sigma_{\mathbf{j}-\mu}^{z}\right)
\end{gathered}
$$


with $t_{1} \equiv \tanh \alpha_{1}$.

Eq.(3.10) yields for the energy $E_{I s i n g, D}^{F P,(1)}$ :

$$
E_{I \operatorname{sing}, D}^{F P,(1)}=-N\left(\cosh \alpha_{1}\right)^{2 D}
$$

and for the $U_{I s i n g, D=1,2}^{F P,(1)}$ interaction Hamiltonians:

$$
\begin{gathered}
U_{I \text { sing }, D=1}^{F P,(1)}=-\left(\cosh \alpha_{1}\right)^{2} \sum_{j=1}^{N}\left(2 t_{1} \sigma_{j}^{z} \sigma_{j+1}^{z}-t_{1}^{2} \sigma_{j}^{z} \sigma_{j+2}^{z}\right) \\
U_{I s i n g, D=2}^{F P,(1)}=-\left(\cosh \alpha_{1}\right)^{4} \sum_{\mathbf{j}}\left[2 t_{1} \sum_{\mu} \sigma_{\mathbf{j}}^{z} \sigma_{\mathbf{j}+\mu}^{z}-t_{1}^{2} \sum_{\mu} \sigma_{\mathbf{j}}^{z} \sigma_{\mathbf{j}+2 \mu}^{z}\right. \\
-2 t_{1}^{2} \sigma_{\mathbf{j}}^{z}\left(\sigma_{\mathbf{j}+\mu_{1}+\mu_{2}}^{z}+\sigma_{\mathbf{j}+\mu_{1}-\mu_{2}}^{z}\right)+t_{1}^{3} \sigma_{\mathbf{j}}^{z}\left(\sigma_{\mathbf{j}+\mu_{1}}^{z} \sigma_{\mathbf{j}-\mu_{1}}^{z} \sigma_{\mathbf{j}+\mu_{2}}^{z}\right. \\
\left.\left.+\sigma_{\mathbf{j}+\mu_{1}}^{z} \sigma_{\mathbf{j}-\mu_{1}}^{z} \sigma_{\mathbf{j}-\mu_{2}}^{z}+\mu_{1} \leftrightarrow \mu_{2}\right)+t_{1}^{4} \sigma_{\mathbf{j}+\mu_{1}}^{z} \sigma_{\mathbf{j}-\mu_{1}}^{z} \sigma_{\mathbf{j}+\mu_{2}}^{z} \sigma_{\mathbf{j}-\mu_{2}}^{z}\right]
\end{gathered}
$$

A glance at eqs. 3.12 ) and (3.13) shows that both $U_{I s i n g, D=1,2}^{F P,(1)}$ contain a term of the form $\sum_{\mathbf{j}, \mu} \sigma_{\mathbf{j}}^{z} \sigma_{\mathbf{j}+\mu}^{z}$. In fact, this is a general property of $U_{\text {Ising,D }}^{F P,(1)}$ in any dimension $D$. Then, if we make the identification:

$$
\lambda=2\left(\cosh \alpha_{1}\right)^{2 D} \tanh \alpha_{1}
$$

we may say that $U_{\text {Ising, } D}$ and $U_{I s i n g, D}^{F P,(1)}$ coincide to order $\lambda$ but differ in order $\lambda^{2}$ and higher. On the other hand, if one eliminates $\alpha_{1}$ in terms of $\lambda$ with the help of (3.14) and substitutes back the result in (3.11), one immediately finds the energy of the state $\psi^{(1)}$. In the $1 D$ case, this energy reads:

$$
E_{I s i n g, D=1}^{F P,(1)}=-\frac{N}{2}\left(1+\sqrt{1+\lambda^{2}}\right)
$$

This expresion agrees to second order in $\lambda$ with the exact result which is given in the termodinamic limit $N \longrightarrow \infty$ by [3]:

$$
e_{\text {Ising }, D=1}=-\lim _{N \rightarrow \infty} \frac{E_{\text {Ising }, D=1}}{N}=(1+\lambda) F\left(-\frac{1}{2}, \frac{1}{2} ; 1 ; \frac{4 \lambda}{(1+\lambda)^{2}}\right)
$$

where $F(a, b ; c ; x)$ is the usual hypergeometric function [24].

From eqs. 3.12) and (3.13) it is clear how to improve the first order aproximation. One has to add to the exponent of the ansatz those terms necessary to cancell the unwanted ones. In the $1 D$ case that corresponds to the choice:

$$
\psi^{(2)}=e^{\frac{1}{2} \sum_{j=1}^{N}\left[\alpha_{1} \sigma_{j}^{z} \sigma_{j+1}^{z}+\alpha_{2} \sigma_{j}^{z} \sigma_{j+2}^{z}\right]}
$$

The parameter $\alpha_{2}$ has been introduced as to eliminate the term $\sum_{j=1}^{N} \sigma_{j}^{z} \sigma_{j+2}^{z}$ appearing in (3.12). Following the same steps as in (3.10) we obtain for the potential $U_{I s i n g, D=1}^{F P,(2)}$ and energy $E_{I s i n g, D=1}^{F P,(2)}$ the equation:

$$
U_{I s i n g, D=1}^{F P,(2)}-E_{I s i n g, D=1}^{F P,(2)}=\left(\cosh \alpha_{1} \cosh \alpha_{2}\right)^{2}
$$




$$
\times \sum_{j=1}^{N}\left(1-t_{1} \sigma_{j}^{z} \sigma_{j+1}^{z}\right)\left(1-t_{1} \sigma_{j}^{z} \sigma_{j-1}^{z}\right)\left(1-t_{2} \sigma_{j}^{z} \sigma_{j+2}^{z}\right)\left(1-t_{2} \sigma_{j}^{z} \sigma_{j-2}^{z}\right)
$$

where

$$
t_{a} \equiv \tanh \alpha_{a}, \quad a=1,2
$$

Now, by demanding $U_{\text {Ising }}$ agrees to order $\lambda^{2}$ with $U_{I s i n g, D=1}^{F P,(2)}$ implies:

$$
\begin{gathered}
E_{I s i n g, D=1}^{F P,(2)}=-N\left(\cosh \alpha_{1} \cosh \alpha_{2}\right)^{2} \\
\lambda=2\left(\cosh \alpha_{1} \cosh \alpha_{2}\right)^{2} t_{1}\left(1-t_{2}\right) \\
0=2 t_{2}-t_{1}^{2}
\end{gathered}
$$

These equations can be easily solved for $E_{I s i n g, D=1}^{F P,(2)}$ as a function of $\lambda$, namely:

$$
E_{I s i n g, D=1}^{F P,(2)}=-N \frac{\lambda}{t_{1}(\lambda)\left(2-t_{1}^{2}(\lambda)\right)}
$$

where $t_{1}(\lambda)$ is the real and positive solution of the polynomial

$$
t_{1}^{4}+t_{1}^{2}+\frac{4 t_{1}}{\lambda}-2=0
$$

In fig.2 a plot of the values for minus the energy density $e_{I \text { sing, } D=1}^{F P,(1)}, e_{I s i n g, D=1}^{F P,(2)}$ and $e_{I s i n g, D=1}$ is presented. Obviously the results improve significantly as we increase the order of the aproximation.

This procedure can be repeated order by order by adding successively terms of more complicated structure in the exponential ansatz. The hope is that after a finite number of steps the process ends up. This is indeed the case, as can be seen for a $1 D$ chain with $N=4$ (see appendix B). We can say that the ground state solution of the Hamiltonian (3.1) can be constructed in a process which is similar to the one used in perturbation theory, but with two basic differences; firstly, it incorporates at each step non perturbative contributions to the energy and to the ground state; and secondly, the process can be completed after a finite number of steps for finite chains.

\section{A Fokker-Planck extension of the Ising model}

We have just described an attempt to reconstruct the exact ground state of a 1dimensional Ising model based in the choice of an exponential ansatz. This is certainly feasible for small chains, as shown in appendix B, but it becomes an awful task for chains already modest in size. On the other hand, it is not our purpose to obtain in a complicated manner what can be easily done by other means [2], [3]. The Ising model in $1 D$ is an integrable system and can be solved by performing a Jordan-Wigner transformation to fermion operators followed by a Bogoliubov transformation. In higher dimensions, however, the model is probably not integrable and that explains the difficulty in finding an exact result, which quite likely does not exist. One of the main interests to seek exact solutions of lattice models consists in 
the study of their critical properties. Thus, if the model under consideration comes from a discretization of a model in the continuum, one would like to recover that theory as the critical point of the corresponding lattice model. In any case, the choice of a particular hamiltonian is not so important as the universality class to which it belongs to. So in this section we propose a FP-hamiltonian which enjoies the same universality properties as the 1-dimensional ITF model, while remaining close to this latter outside the critical region, in the sense that the energy and other physical quantities do not deviate significantly.

Let us start by considering the most general 1-dimensional ITF hamiltonian including all possible couplings involving only two $\sigma^{z}$ matrices, namely:

$$
H_{\text {Ising }}\left(\lambda_{1}, \cdots, \lambda_{N}\right)=-\sum_{j=1}^{N} \sigma_{j}^{x}-\frac{1}{2} \sum_{j=1}^{N} \sum_{r=1}^{N-1} \lambda_{r} \sigma_{j}^{z} \sigma_{j+r}^{z}
$$

where, for consistency:

$$
\lambda_{r}=\lambda_{N-r} \quad, \quad r=1, \cdots, N-1
$$

Notice that the standard 1-dimensional ITF model corresponds to the choice: $\lambda_{1}=\lambda_{N-1}=\lambda$ and $\lambda_{r}=0$ for $r \neq 1$ or $N-1$.

We want to find a FP hamiltonian which coincides with (4.1) except for operators involving more than two $\sigma^{z}$-matrices, i.e:

$$
U_{I s i n g}^{F P}\left(\lambda_{1}, \cdots, \lambda_{N}\right)-U_{\text {Ising }}\left(\lambda_{1}, \cdots, \lambda_{N}\right)=\sum_{p \geq 3} \sum_{i_{1}, \cdots, i_{p}} C_{i_{1}, \cdots, i_{p}} \sigma_{i_{1}}^{z} \cdots \sigma_{i_{p}}^{z}
$$

According to the FP-philosophy, the knowledge of $H_{I s i n g}^{F P}$ is equivalent to the knowledge of its ground state, which we shall suppose to be given by:

$$
\left|\psi\left(\alpha_{1}, \cdots, \alpha_{N}\right)\right\rangle=e^{-\frac{1}{2} S\left(\sigma_{1}^{z}, \cdots, \sigma_{N}^{z}\right)}|0\rangle=e^{\frac{1}{4} \sum_{r=1}^{N-1} \sum_{j=1}^{N} \alpha_{r} \sigma_{j}^{z} \sigma_{j+r}^{z}}|0\rangle
$$

where $\alpha_{r}=\alpha_{N-r}$ are assumed to be real and $|0\rangle$ satisfies (3.3).

The discrete Ricatti equation reads for the ansatz (4.4):

$$
\begin{gathered}
E^{F P}+\frac{1}{2} \sum_{j=1}^{N} \sum_{r=1}^{N-1} \lambda_{r} \sigma_{j}^{z} \sigma_{j+r}^{z} \\
-\sum_{p \geq 3} \sum_{i_{1}, \cdots, i_{p}} C_{i_{1}, \cdots, i_{p}} \sigma_{i_{1}}^{z} \cdots \sigma_{i_{p}}^{z}=-\sum_{j=1}^{N} e^{\Delta_{j}(S)}
\end{gathered}
$$

where

$$
\Delta_{j}(S)=-\sum_{r=1}^{N-1} \alpha_{r} \sigma_{j}^{z} \sigma_{j+r}^{z}
$$

The $\sigma_{j}^{z}$ operators in eq.4.5) can in fact be replaced by variables $\sigma_{j}$ taking just two values \pm 1 . Then if we multiply both sides of (4.5) by a convenient number of $\sigma_{j}$ 's and subsequently we sum over the $2^{N}$ possible choices of $\sigma_{j}$ 's we obtain for the energy $E^{F P}$ and the coupling constants $\lambda_{r}$ 


$$
\begin{gathered}
E^{F P}=-\frac{N}{2^{N}} \sum_{\left\{\sigma_{1}, \cdots, \sigma_{N}\right\}} e^{\Delta_{0}(S)} \\
\lambda_{r}=-\frac{1}{2^{N}} \sum_{\left\{\sigma_{1}, \cdots, \sigma_{N}\right\}}\left(\sum_{j=1}^{N} \sigma_{j} \sigma_{j+r}\right) e^{\Delta_{0}(S)}
\end{gathered}
$$

Here the subindex 0 in $\Delta_{0}(S)$ refers to the site " $0 "$ " (= site " $N$ ") of the chain. It is straightforward to prove that

$$
e^{\Delta_{0}(S)}=\prod_{r=1}^{N-1} \cosh \alpha_{r}\left(1-t_{r} \sigma_{0} \sigma_{r}\right)
$$

where $t_{r} \equiv \tanh \alpha_{r}$. If we now introduce (4.9) in (4.7)-(4.8) and define:

$$
1=-t_{0}
$$

we obtain:

$$
\begin{gathered}
e_{N}^{F P} \equiv \frac{-E^{F P}}{N}=\prod_{r=1}^{N-1} \cosh \alpha_{r} \\
\frac{\lambda_{r}}{e_{N}^{F P}}=-\sum_{n=0}^{N-1} t_{n} t_{r-n} \quad, \quad(r=1, \cdots, N-1)
\end{gathered}
$$

where $e_{N}^{F P}$ is minus the FP-energy density of a chain of length $N$.

The structure of eqs.(4.11)-(4.12) has already emerged in, for example, eq.(3.20) (see also eqs. (B.13)-(B.14) of appendix B). These equations relate the $t_{n}$ (or the $\left.\alpha_{n}\right)$ to the coupling constants $\lambda_{r}$ and, therefore, one could in principle relate the FP-energy $E^{F P}$ with the $\lambda$ 's. In this whole process, the parameters $C_{i_{1}, \cdots, i_{p}}$ which stablish the difference between our model and the pure Ising model (4.1) play no role. In fact, they can be computed a posteriori in terms of the $\alpha$ 's. This means that our prescription to associate a FP-model to the Ising is well defined.

The next step is the resolution of eqs.(4.11)-(4.12). For that, let us introduce the Fourier transformed $\hat{t}_{n}$ of the $t_{n}$ :

$$
t_{n}=\frac{1}{\sqrt{N}} \sum_{m=0}^{N-1} e^{\frac{2 \pi i n m}{N}} \hat{t}_{m}
$$

and consequently eq.(4.12) becomes:

$$
\frac{\lambda_{r}}{e_{N}^{F P}}=-\sum_{m=0}^{N-1} e^{\frac{2 \pi i r m}{N}}\left(\hat{t}_{m}\right)^{2}
$$

Then, if we apply an inverse Fourier transformation to (4.14) we get the $\hat{t}_{n}$ 's in terms of the $\lambda$ 's and a parameter $C_{N}$, namely:

$$
\hat{t}_{m}=-\frac{1}{\sqrt{N}} \varepsilon_{m}\left(C_{N}-\sum_{r=1}^{N-1} e^{\frac{-2 \pi i r m}{N}} \frac{\lambda_{r}}{e_{N}^{F P}}\right)^{1 / 2}
$$

where $\varepsilon_{m}=\varepsilon_{N-m}= \pm 1$ and 


$$
C_{N}=\sum_{m=0}^{N-1}\left(\hat{t}_{m}\right)^{2}=\sum_{n=0}^{N-1} t_{n}^{2}
$$

is a real constant which can be fixed by imposing the eq. (4.10):

$$
1=-\frac{1}{\sqrt{N}} \sum_{m=0}^{N-1} \hat{t}_{m}=\frac{1}{N} \sum_{m=0}^{N-1} \varepsilon_{m}\left(C_{N}-\sum_{r=1}^{N-1} e^{\frac{-2 \pi i r m}{N}} \frac{\lambda_{r}}{e_{N}^{F P}}\right)^{1 / 2}
$$

One can easily realize that the trivial solution for the case $\lambda_{r}=0, \forall r$, corresponds to:

$$
\begin{gathered}
t_{n}=-\delta_{n, 0} \quad, \quad \hat{t}_{m}=-\frac{1}{\sqrt{N}} \\
C_{N}=1 \quad, \quad \varepsilon_{m}=1
\end{gathered}
$$

The presence of interaction modifies these values; for convenience, however, we shall continue maintaining $\varepsilon_{m}=1$.

When $\lambda=\lambda_{1}=\lambda_{N-1},\left(\lambda_{r}=0\right.$, otherwise $)$, one recovers the usual ITF model (3.1). Eqs.(4.13) and (4.17) becomes now:

$$
\begin{gathered}
t_{n}=-\frac{1}{N} \sum_{m=0}^{N-1} e^{\frac{2 \pi i n m}{N}}\left(C_{N}-\frac{2 \lambda}{e_{N}^{F P}} \cos \left(\frac{2 \pi m}{N}\right)\right)^{1 / 2} \\
1=\frac{1}{N} \sum_{m=0}^{N-1}\left(C_{N}-\frac{2 \lambda}{e_{N}^{F P}} \cos \left(\frac{2 \pi m}{N}\right)\right)^{1 / 2}
\end{gathered}
$$

Notice that (4.20) is nothing but the $n=0$ case of (4.19).

We shall look for solutions of (4.19) and (4.20) for which the $t_{n}$ are real quantities. This is guaranted if we impose the constraint:

$$
0 \leq u_{N} \equiv \frac{2 \lambda}{C_{N} e_{N}^{F P}} \leq 1
$$

The solution of (4.19) and (4.20) involves, in principle, four steps: i) to express $C_{N}$ as a function of $\lambda$ and $e_{N}^{F P}$, ii) to rewrite $t_{n}$ in terms of $\lambda$ and $e_{N}^{F P}$, iii) to introduce the $t_{n}$ in eq.(4.11) as to obtain an equation for $e_{N}^{F P}$ as a function of $\lambda$, and iv) to solve this equation.

Fortunately, this rather complicated elimination process can be explicitely accomplished in two extreme cases according as: a) $u_{N}=1$ and $N$ arbitrary or b) $u_{N}<1$ but $N \longrightarrow \infty$. Next we shall describe our results leaving the details and derivations to the Appendix C.

a) Case $u_{N}=1$

The values of $t_{n}$ and $e_{N}^{F P}$ are given by:

$$
\begin{gathered}
t_{n}=\frac{\left(\sin \frac{\pi}{2 N}\right)^{2}}{\sin \frac{\pi}{N}\left(n+\frac{1}{2}\right) \sin \frac{\pi}{N}\left(n-\frac{1}{2}\right)} \\
e_{N}^{F P}=\frac{\sqrt{2}}{N \sin \frac{\pi}{2 N} \sin \frac{N \theta_{N}}{2}}
\end{gathered}
$$


where the angle $\theta_{N}$ in (4.23) is defined as:

$$
\cos \theta_{N}=2 \cos \left(\frac{\pi}{N}\right)-1
$$

If we take the limit $N \longrightarrow \infty$ in eqs.(4.22) and (4.23) we deduce that the model exhibits critical behaviour. First of all, from eq. (4.22) we obtain

$$
\lim _{N \rightarrow \infty} t_{n}=\frac{1}{4 n^{2}-1}
$$

and hence for $n$ large the "amplitudes" $t_{n}$ have a scaling behaviour. It is curious to observe that the value of $t_{n}$ given by eq. (4.25) coincides with the correlator of two $\sigma^{x}$ 's matrices in the standard ITF model at the critical point [3]:

$$
\left\langle\sigma_{j}^{x} \sigma_{j+n}^{x}\right\rangle-\left\langle\sigma_{j}^{x}\right\rangle^{2}=\left(\frac{2}{\pi}\right)^{2} \frac{1}{4 n^{2}-1}
$$

We may wonder whether these two results are related.

Another indication of criticality arises from the $1 / N^{2}$ correction to the ground state energy density, which is given by:

$$
e_{N}^{F P}=e_{\infty}^{F P}\left[1+\frac{\pi^{2}}{24 N^{2}}\left(1-\frac{\pi}{\sqrt{2}} \cot \left(\frac{\pi}{\sqrt{2}}\right)\right)+O\left(\frac{1}{N^{3}}\right)\right]
$$

where

$$
e_{\infty}^{F P}=\frac{2 \sqrt{2}}{\pi \sin (\pi / \sqrt{2})} \cong \frac{3.554}{\pi}
$$

Recall that $e_{N}^{F P}$ is minus the energy density of the ground state.

It is well known that in a $1 D$ conformal invariant theory this correction satisfies the Cardy formula:

$$
e=e_{\infty}+\frac{\pi c v_{s}}{6 N^{2}}
$$

where $v_{s}$ is defined through the dispersion relation of the elementary excitation above the ground state, i.e. $\omega(k) \approx v_{s} k$, and $c$ is the central extension of the Virasoro algebra underlying the corresponding conformal field theory.

Compairing eqs. (4.27) and (4.29) we learn

$$
c v_{s}=\frac{\pi}{4} e_{\infty}^{F P}\left(1-\frac{\pi}{\sqrt{2}} \cot \left(\frac{\pi}{\sqrt{2}}\right)\right)
$$

Hence we would need to know the value of $v_{s}$ in order to derive the central extension $c$ (see section 5 for some considerations concerning this point). Our expectations are that $c=\frac{1}{2}$, in which case we would be actually describing an Ising model; but we shall arrive to this result by studying other quantities, such as the critical exponents.

It is amusing to observe that the value of the critical energy density (4.28) is comparable with the one of the ITF model: 


$$
e_{\infty}^{I T F}=\frac{4}{\pi}
$$

To complete this comparison, let us recall that in 1D the critical value of $\lambda$ is given by $\lambda_{c}^{I T F}=1$, whereas in our FP-ITF model we find

$$
\lambda_{c}^{F P}=\frac{\pi}{4 \sqrt{2} \sin (\pi / \sqrt{2})} \cong 0.6979
$$

b) Case $u_{\infty}<1$

From the discussion of the previous paragraph we expect that this case will account for the non critical regime of the model. The expressions for the $t_{n}$ and the energy $e_{\infty}^{F P}$ are given here by (see Appendix $\mathrm{C}$ for computation details ):

$$
\begin{gathered}
t_{n}=\frac{1}{\pi 2^{5 / 2}}\left[\frac{2 \lambda_{R}}{\left(1+\lambda_{R}\right)^{2}}\right]^{n} \frac{\Gamma\left(\frac{n}{2}-\frac{1}{4}\right) \Gamma\left(\frac{n}{2}+\frac{1}{4}\right)}{\Gamma(n+1)} \frac{F\left(n-\frac{1}{2}, n+\frac{1}{2}, 2 n+1 ; \frac{4 \lambda_{R}}{\left(1+\lambda_{R}\right)^{2}}\right)}{F\left(-\frac{1}{2}, \frac{1}{2}, 1 ; \frac{4 \lambda_{R}}{\left(1+\lambda_{R}\right)^{2}}\right)} \\
e_{\infty}^{F P}=\frac{\lambda}{\lambda_{R}}\left[\left(1+\lambda_{R}\right) F\left(-\frac{1}{2}, \frac{1}{2}, 1 ; \frac{4 \lambda_{R}}{\left.\left(1+\lambda_{R}\right)^{2}\right)}\right]^{2}\right.
\end{gathered}
$$

where we have introduced a new variable $\lambda_{R}$ through:

$$
u_{\infty}=\frac{2 \lambda_{R}}{1+\lambda_{R}^{2}}
$$

Equations (4.33) and (4.34) make clear that $\lambda_{R}$ rather than $\lambda$ is the effective coupling constant which governs the behaviour of the ground state. In a certain sense, $\lambda_{R}$ results from the dressing of the "bare" coupling constant $\lambda$ by all the interactions present in the FP-Hamiltonian. The explicit form of $\lambda$ as a function of $\lambda_{R}$ is very complicated and it will not be needed; in any case it can be obtained by comparing eq.(4.35) with the eq. (C.18) of Appendix $\mathrm{C}$ for $e_{\infty}^{F P}$ as a function of the $t_{n}$ 's. In fact, it is not difficult to show that

$$
\lambda=\lambda_{R}\left(1-\frac{1}{2} \lambda_{R}^{2}+O\left(\lambda_{R}^{4}\right)\right)
$$

and

$$
\lim _{\lambda_{R} \rightarrow 1}\left(\frac{d \lambda}{d \lambda_{R}}\right)=0
$$

Making use of (4.37) one sees that $\frac{\partial e_{\infty}^{F P}}{\partial \lambda_{R}}$ is not singular at the critical value $\lambda_{R}=1$. However, the "specific heat" defined as the second derivative $\frac{\partial^{2} e_{\infty}^{F P}}{\partial \lambda_{R}{ }^{2}}$ does actually exhibit a logarithm divergence

$$
\frac{\partial^{2} e_{\infty}^{F P}}{\partial \lambda_{R}{ }^{2}} \simeq-\frac{8 \lambda_{c}}{\pi^{2}} \log \left(1-\lambda_{R}\right)
$$


This is precisely the same logarithm singular behaviour that one would expect from the original ITF model [3]. This implies that the critical exponent $\alpha$ defined through $\frac{\partial^{2} e}{\partial \lambda^{2}} \simeq(1-\lambda)^{\alpha}$ is the same for the two models, i.e. $\alpha=0$.

Let us concentrate now on the behaviour of the $t_{n}$ for $n$ large. In the critical case $\lambda_{R}=1$, we have seen that they satisfy the scaling law (4.25); if $\lambda_{R}<1$ we obtain instead

$$
t_{n} \sim \frac{1}{n^{3 / 2}} \lambda_{R}^{n} \quad \text { for } n>>1, \quad \lambda_{R}<1
$$

and so eq.(4.39) allows one to define a correlation length $\xi\left(\lambda_{R}\right)$ through $t_{n} \sim \frac{1}{n^{3 / 2}} e^{-\frac{n}{\xi}}$ as

$$
\xi^{-1}\left(\lambda_{R}\right)=-\log \lambda_{R} \simeq\left(1-\lambda_{R}\right), \quad \lambda_{R} \simeq 1
$$

which implies in particular that the critical exponent $\nu$ is equal to 1 , just like in the standard ITF model. This is again another indication that the model we are considering belongs to the same universality class as the standard ITF model.

\section{A change of variables: excited states}

Until now we have considered the construction of the ground state of a lattice Hamiltonian in the spirit of the FP procedure; at this point, it seems natural to wonder what can tell us the method about the rest of the eigenstates of the Hamiltonian. This is, of course, important both for the study of gaps in the spectrum as for the dispersion relation of elementary excitations.

In order to fix ideas, let us start by posing the problem in the context of Quantum Mechanics. Hitherto, we have been using the fact that a wave function of the form $\psi_{0}(x)=e^{-S(x)}, x \in \mathbf{R}$, is a ground state of the FP-Hamiltonian:

$$
H^{F P}=\frac{1}{2}\left[-\frac{d^{2}}{d x^{2}}+\left(\frac{d S}{d x}\right)^{2}-\frac{d^{2} S}{d x^{2}}\right]
$$

If, for example, we take $S(x)=\frac{1}{2} x^{2}$, then $H^{F P}$ coincides with the number operator for the harmonic oscillator $\left(H^{F P}=a^{\dagger} a=\mathcal{N},\left[a, a^{\dagger}\right]=1\right)$. If we want to study the excited states of (5.1), we could make the ansatz: $\psi(x)=A(x) e^{-S(x)}$, which can be thought of as a change of variables from $\psi(x)$ to $A(x)$ since the function $S(x)$ is known a priori. The eigenvalue problem for $\psi(x)$ becomes then:

$$
H^{F P} \psi(x)=\Delta E \psi(x) \quad \Longrightarrow \quad H^{R F P} A(x)=\Delta E A(x)
$$

where

$$
H^{R F P}=\frac{1}{2}\left[-\frac{d^{2}}{d x^{2}}+2 \frac{d S}{d x} \frac{d}{d x}\right]
$$

and $\Delta E$ represents the difference of energies between the excited state $\psi(x)$ and the ground state $\psi_{0}(x)$-whose energy is actually zero. We shall call $H_{R F P}$ the reduced Fokker-Planck Hamiltonian. The ground state solution using the new variable $A(x)$ corresponds to $A(x)=C t e$. In particular, for the harmonic oscillator, eq.(5.2) reads: 


$$
-\frac{1}{2} \frac{d^{2} A}{d x^{2}}+x \frac{d A}{d x}=\Delta E A
$$

which becomes a confluent hypergeometric equation after the substitution $y=x^{2}$, and whose normalized solutions are given by the well-known Hermite polynomials. It is worth noticing that $H^{R F P}$ is not an hermitean operator with respect to the measure $\mu=d x$, but it is so for the measure $\mu=e^{-2 S(x)} d x$.

Next, we shall try to generalize the previous methods to more complicated Hamiltonians. More precisely, the problem we face is the construction of the spectrum of the FP-Hamiltonian associated to (4.1), namely:

$$
H^{F P}=-\sum_{j=1}^{N}\left[\sigma_{j}^{x}-e^{\Delta_{j}(S)}\right]=-\sum_{j=1}^{N}\left[\sigma_{j}^{x}-e_{N}^{F P} \prod_{r=1}^{N-1}\left(1-t_{r} \sigma_{j}^{z} \sigma_{j+r}^{z}\right)\right]
$$

whose ground state $\psi_{0}$ was defined in (4.4):

$$
\left|\psi_{0}\right\rangle=\exp \left(\frac{1}{4} \sum_{r=1}^{N-1} \sum_{j=1}^{N} \alpha_{r} \sigma_{j}^{z} \sigma_{j+r}^{z}\right)|0\rangle
$$

In (5.5) $e_{N}^{F P}$ represents minus the FP-energy density given by (4.11) and $t_{r} \equiv$ $\tanh \alpha_{r}$. In principle, this ground state may not be unique. For example, this would happen if the Hamiltonian (5.5) enters into an ordered region characterized by ground states with opposite non-vanishing magnetization. We shall not discuss this possibility here.

The change of variables adequate for our purposes corresponds to:

$$
|\psi\rangle=A\left(\sigma_{1}^{z}, \cdots, \sigma_{N}^{z}\right)\left|\psi_{0}\right\rangle
$$

where $A\left(\sigma_{1}^{z}, \cdots, \sigma_{N}^{z}\right)$ is the most general operator involving $\sigma^{z}$ 's. With the notations of appendix B:

$$
A\left(\sigma_{1}^{z}, \cdots, \sigma_{N}^{z}\right)=\sum_{a=1}^{2^{N}} A_{I_{a}} \sigma_{I_{a}}^{z}
$$

where in contrast to the appendix, we shall allow in $I_{a}$ the presence of any number of 1's, independently of their parity. The eigenvalue equation (5.2) reads now:

$$
H^{R F P}|A\rangle=\Delta E|A\rangle
$$

where

$$
|A\rangle=A\left(\sigma_{1}^{z}, \cdots, \sigma_{N}^{z}\right)|0\rangle \equiv \sum_{a=1}^{2^{N}} A_{I_{a}}\left|I_{a}\right\rangle
$$

and

$$
H^{R F P}=\sum_{j=1}^{N} e^{\Delta_{j}(s)}\left(\mathbf{1}-\sigma_{j}^{x}\right)=e_{N}^{F P} \sum_{j=1}^{N} \prod_{r=1}^{N-1}\left(\mathbf{1}-t_{r} \sigma_{j}^{z} \sigma_{j+r}^{z}\right)\left(\mathbf{1}-\sigma_{j}^{x}\right)
$$


For reasons will be evident latter, it will be of some convenience for us to introduce the parameter:

$$
\varepsilon \equiv \frac{\Delta E}{2 e_{N}^{F P}}
$$

and in what follows we will also assume that $H^{R F P}$ is divided by $2 e_{N}^{F P}$ to give $\hat{H}^{R F P}$. Notice that in the absence of interaction (i.e $t_{r}=0, \forall r$ ), we have:

$$
\hat{H}^{(0)} R F P=\sum_{j=1}^{n} \frac{\mathbf{1}-\sigma_{j}^{x}}{2} \equiv \mathcal{N}
$$

and so $\hat{H}^{0 R F P}$ coincides with the number operator $\mathcal{N}$, i.e, the operator which in this context counts the number $n_{I_{a}}$ of 1 's of a given state $\left|I_{a}\right\rangle$ :

$$
\mathcal{N}\left|I_{a}\right\rangle=n_{I_{a}}\left|I_{a}\right\rangle
$$

An interesting property of the operator $\hat{H}^{R F P}$ is

$$
\sum_{a=1}^{2^{N}}\left\langle I_{a}\left|\hat{H}^{R F P}\right| I_{b}\right\rangle=\prod_{r=1}^{N-1}\left(1-t_{r}\right) n_{I_{b}}
$$

This equation suggests some kind of connection between the evolution described by the Hamiltonian $\hat{H}^{R F P}$ and a Markov process. As a consequence of (5.15) we find

$$
\varepsilon=\prod_{r=1}^{N-1}\left(1-t_{r}\right) \frac{\sum_{a=1}^{2^{N}} n_{I_{a}} A_{I_{a}}}{\sum_{a=1}^{2^{N}} A_{I_{a}}}
$$

A useful way of reexpressing $\hat{H}^{R F P}$ is

$$
\begin{gathered}
\hat{H}^{R F P}\left(\left\{t_{r}\right\}\right)=-\sum_{r=0}^{N-1} t_{r} \Lambda_{r}+\sum_{1 \leq r_{1}<r_{2} \leq N-1} t_{r_{1}} t_{r_{2}} \Lambda_{r_{1}, r_{2}}+\cdots \\
\cdots+(-1)^{l} \sum_{\substack{1 \leq r_{1}<r_{2}<\cdots r_{l} \leq N-1\\
}} t_{r_{1}} \cdots t_{r_{l}} \Lambda_{r_{1}, \cdots, r_{l}}+\cdots \\
-t_{1} t_{2} \cdots t_{N-1} \Lambda_{1,2, \cdots, N-1}
\end{gathered}
$$

where the operators $\Lambda$ 's are defined as

$$
\Lambda_{r_{1}, \cdots, r_{l}}=\sum_{j=1}^{N}\left(\sigma_{j}^{z}\right)^{l} \sigma_{j+r_{1}}^{z} \cdots \sigma_{j+r_{l}}^{z} \frac{1-\sigma_{j}^{x}}{2} \quad, \quad \Lambda_{0} \equiv \mathcal{N}
$$

Notice that the first sum in (5.17) starts at $r=0$ and so reproduce the operator $\mathcal{N}$ (recall that $t_{0}=-1$ ).

The action of $\Lambda$ on a generic state $\left|I_{a}\right\rangle=\left|\mu_{1}, \cdots, \mu_{N}\right\rangle,\left(\mu_{i}=0\right.$ or $\left.1, \bmod (2)\right)$ is given by (see fig.3):

$$
\Lambda_{r_{1}, \cdots, r_{l}}\left|\mu_{1}, \cdots, \mu_{N}\right\rangle=\sum_{j=1}^{N} \delta_{1, \mu_{j}}\left|\cdots, \mu_{j}+l, \cdots, \mu_{j+r_{1}}+1, \cdots, \mu_{j+r_{l}}+1, \cdots\right\rangle
$$


The diagonalization of the Hamiltonian (5.17) seems, a priori, not much easier than the diagonalization of the Hamiltonian (5.5). However, we have developed a real renormalization group approach which enables us to deal with this apparent difficulty; but the results concerning this point will be presented elsewhere [25]. For the moment, and with the purpose of getting some insight into the Hamiltonian (5.17), let us consider only the first term in it which is linear in the $t$ 's, namely:

$$
\hat{H}^{(1) R F P}=-\sum_{r=0}^{N-1} t_{r} \Lambda_{r}
$$

It is easy to see then that the state:

$$
\left|\psi_{m}\right\rangle=\sum_{j=1}^{N} e^{2 \pi i m j / N}|j\rangle
$$

where $|j\rangle=\left|0 \cdots 0{ }_{1}^{j} 0 \cdots 0\right\rangle$ is an eigenstate of $\hat{H}^{(1) R F P}$ :

$$
\hat{H}^{(1) R F P}\left|\psi_{m}\right\rangle=-\sqrt{N} \hat{t}_{m}\left|\psi_{m}\right\rangle
$$

Hence, eq.(4.15) can be used to obtain the dispersion relation for a plane wave of momentum $k=\frac{2 \pi m}{N}$

$$
\Delta E^{F P}(k)=2 e_{N}^{F P}\left(C-\frac{2 \lambda \cos k}{e_{N}^{F P}}\right)^{1 / 2}
$$

The gap is obtained for $k=0$ and vanishes in the critical point, i.e when $u_{N}=1$. Moreover, for $k$ small the dispersion relation (5.23) is linear in $k$ :

$$
\Delta E^{F P}(k) \simeq \frac{\pi}{2} e_{\infty}^{F P} k
$$

whence we deduce a speed of sound $v_{s}^{(1)}=\frac{\pi}{2} e_{\infty}^{F P}$. This confirms our expectations that the value $u_{N}=1$ actually corresponds to a critical point. Unfortunately, the speed of sound we derive from eq.(5.24) is not the speed of sound of the excitations of the whole Hamiltonian (5.17). In fact if we take for granted that $c=1 / 2$ is the value of the central extension of this model, then the speed of sound for the Hamiltonian (5.20) should be given by:

$$
v_{s}^{(1)}=\frac{\pi}{2} e_{\infty}^{F P}\left(1-\frac{\pi}{\sqrt{2}} \cot \left(\frac{\pi}{\sqrt{2}}\right)\right)
$$

This equation suggests that the role of the additional terms in the Hamiltonian (5.17) is simply to redefine the value of $v_{s}^{(1)}$.

In section 4 we introduced an effective coupling constant $\lambda_{R}$ in terms of which the energy $e_{\infty}^{F P}$ displayed a critical behaviour. If we now rewrite eq.(5.23) as a function of $\lambda_{R}$ we obtain

$$
\Delta E^{F P}(k)=2 \sqrt{\frac{\lambda e_{\infty}^{F P}}{\lambda_{R}}}\left(1+\lambda_{R}^{2}-2 \lambda_{R} \cos k\right)^{1 / 2}
$$

It is noteworthy to compare this relation with the exact dispersion relation of the standard ITF model. As it is shown in [3] 


$$
\Delta E_{I T F}^{\text {exact }}(k)=2\left(1+\lambda^{2}-2 \lambda \cos k\right)^{1 / 2}
$$

The evident similarity between eqs.(5.26) and (5.27) is again another confirmation that the model we are describing is very close to the usual Ising model; indeed, we believe they are identical at the critical point.

\section{Conclusions and final remarks}

This paper represents a first step in the application of the Fokker-Planck techniques to the study of quantum lattice Hamiltonians. The main idea is to approximate the Hamiltonian of interest $H$ by a FP-Hamiltonian $H^{F P}$ which shares with $H$ a certain number of properties. Firstly, we have considered FP-Hamiltonians which agree with $H$ to first and second order in perturbation theory. Next, and more interesting, we have built up a FP-Hamiltonian belonging to the same universality class as $H$. In both cases the advantage of working with a FP-Hamiltonian is that we know the exact ground state of this latter. In the example we have considered, i.e. the ITF model, we have been able to solve the equations fixing the ground state parameters in terms of the coupling constant, and that makes feasible the study of the critical behaviour of the model.

Below we list some issues we believe deserve further investigation.

\section{Modelistic:}

- The Ising model for $D>1$. Sections 4, 5 and 6 can automatically be extended to ITF models in higher dimensions. As a matter of fact, increasing the space dimension does not represent a priory a barrier to the FP-techniques, as is shown in appendix D. Our feeling is, however, that realistic results would require of exponential ansatz involving more than two $\sigma^{z}$ operators. In this sense, the analysis of the $D=2$ ITF model still remains as a challenge in view of its connection with the $3 D$ classical Ising model.

- $\mathbf{Z}_{\mathbf{N}}$-gauge models in $D=2$ and 3. Here we can inmediately apply the FPprocedure developed in the paper to construct the ground state in the confining phase; in $2 D$ this phase is dual to the ordered phase of the $2 D$ ITF model [26].

- The Potts model. FP general machinery of sections 2 can be easily applied to the case of the 1D Potts model [25]. The Potts model is a fruitful generalization of the Ising model and has been received much attention in the context of statistical mechanics. In the notation of section 2 , the $\mathrm{k}$-state $1 D$ Potts Hamiltonian is defined by:

$$
\begin{gathered}
H_{\text {Potts }}(\lambda)=H_{0}+\lambda U_{\text {Potts }}\left(Z_{1}, \cdots, Z_{N}\right) \\
=-\sum_{n=1}^{k-1} \sum_{j=1}^{N} X_{j}^{n}-\lambda \sum_{n=1}^{k-1} \sum_{j=1}^{N}\left(Z_{j} Z_{j+1}^{\dagger}\right)^{n}
\end{gathered}
$$


where the operators $X$ and $Z$ verify $X Z=e^{\frac{2 \pi i}{k}} Z X$ and $X Z^{\dagger}=e^{-\frac{2 \pi i}{k}} Z^{\dagger} X$. The Hamiltonian (6.1) constitutes an example of a traslational $\mathbf{Z}_{k}$ invariant and hermitic operator, which includes the Ising Hamiltonian as a particular case $(k=2)$.

- Fermion models and Heisenberg models. In the example considered in this paper, the operators $V_{I}$ entering in the exponential of the ansatz commuted among themselves. This property facilitated a lot the computations. However, this is not the case for models with fermions or spins [27]. Hence, the question of a convenient implementation of the FP-techiques so as to include fermions still remains to be done.

- Degenerate ground states. The general FP-construction introduced in section 2 applies also when the unperturbed hamiltonian $H_{0}$ has degenerated ground states, as is the case of the ITF model in the ordered phase; here the computation goes in parallel to the well-known degenerated perturbation theory of Quantum Mechanics.

\section{Universality:}

- Apart from the critical exponent $\alpha$ and $\nu$ computed in this paper, one would like to determine the rest of exponents, specially the fractional ones. In fact, only after this determination had been accomplished, could a complete agreement with the critical Ising model be claimed.

- If our statement concerning the universality class of the FP-Hamiltonians is correct, then we have a method to compute the exact values of critical exponents. One may wonder how the scenario would be like in dimensions higher than one, where non trivial integrable models do not exist.

- FP-procedure seems to throw a light on the way we could compute the correlation functions of some operators. For that purpose, we could use the ground state energy as a generating function of correlations along the lines of the Hellmann-Feynman theorem.

- A fundamental question concerning the FP-aproach undertaken in this paper is how to find a "minimal FP-ansatz" capable of reproducing the same critical behaviour of the original Hamiltonian $H$. We do not have a neat answer to this question yet; however common sense recommends to begin with "simple" ansatzs and from these to construct more complicated ones. The final objective must be, of course, to capture the essential physics of the model under consideration.

\section{Integrability:}

- An interesting open question is whether the FP-Hamiltonians are integrable or not, since this would explain some of the magic these Hamiltonians enjoy. If so, one would like to know whether or not there exists a kind of Yang-Baxter equation underlying or responsable for such a behaviour. 


\section{Acknowledgments}

We would like to thank E. Brezin, J.G. Carmona, J.G. Esteve, C. Gómez, M.Halpern, M.A. Martin-Delgado and M.A.H. Vozmediano for useful discussions. This work has been partially supported by the CICYT grant PB92-1092 (G.S.). 


\section{A A q-deformation of the FP-quantum mechan- ical Hamiltonians}

In Section 2 we were concerned with a Fokker-Planck approach to Hamiltonians which appear in continuous or lattice Quantum Field Theories. We devote this appendix to show that most of the formulae appearing there have an analogue in ordinary Quantum Mechanics. For pedagogical reasons, let us concentrate on $1 D$.

To begin with, let us write down the analogous to eqs. (2.3), (2.4) and (2.5) for a wave function $\psi(x)=e^{-S(x)}, x \in \mathbf{R}$ :

$$
\begin{gathered}
H^{F P}=\frac{1}{2}\left[-\frac{d^{2}}{d x^{2}}+\left(\frac{d S}{d x}\right)^{2}-\frac{d^{2} S}{d x^{2}}\right] \\
=\frac{1}{2} R^{\dagger} R \\
R=i \frac{d}{d x}+i \frac{d S}{d x}
\end{gathered}
$$

Then if we put $H^{F P}=H_{0}+U^{F P}(x)-E^{F P}$, we obtain the Ricatti equation

$$
U^{F P}(x)-E^{F P}=\frac{1}{2}\left[\left(\frac{d S}{d x}\right)^{2}-\frac{d^{2} S}{d x^{2}}\right]
$$

Now suppose that $x \equiv \theta$ is compactified on a circle of radius 1 . Then it makes sense to work with the variables $Z=e^{i \theta}$ and rewrite the wave function $\psi(\theta)$ as a single valued function of $Z$, i.e $\psi(Z)$. Similarly, instead of working with the usual momentum $p=-i \hbar \frac{d}{d \theta}$, we may choose the operator $X=e^{i p}$. Both $X$ and $Z$ satisfy commutation relations

$$
X Z=q Z X \quad, \quad q=e^{i \hbar}
$$

Now we define the so-called $q$-derivative $D_{q}$

$$
D_{q}=i \frac{X-X^{-1}}{q-q^{-1}}
$$

which coincides with the usual $\frac{d}{d x}$ in the limit $\hbar \longrightarrow 0$. The free Hamiltonian $H_{0}=-\frac{1}{2} \frac{d^{2}}{d \theta^{2}}$ can therefore be replaced by a " $q$-deformed version":

$$
H_{0}(q)=-\frac{1}{2} D_{q}^{2}=\frac{1}{2\left(q-q^{-1}\right)^{2}}\left(X^{2}+X^{-2}-2\right)
$$

and the corresponding FP-Hamiltonian becomes:

$$
\begin{gathered}
H^{F P}(q)=\frac{1}{2\left(q-q^{-1}\right)^{2}}\left[X^{2}+X^{-2}-e^{S(Z)-S\left(q^{2} Z\right)}-e^{S(Z)-S\left(q^{-2} Z\right)}\right] \\
=\frac{1}{2} R^{\dagger}(q) R(q)
\end{gathered}
$$

with 


$$
R(q)=\frac{i}{q-q^{-1}}\left[e^{\frac{1}{2}\left[S\left(q^{2} Z\right)-S(Z)\right]} X^{2}-e^{-\frac{1}{2}\left[S\left(q^{2} Z\right)-S(Z)\right]}\right]
$$

where the hermiticity of $S$ has implicitely been assumed.

Finally, if we write $H^{F P}(q)$ as $H_{0}(q)+U^{F P}(Z)-E^{F P}$, we get

$$
U^{F P}(Z)-E^{F P}=\frac{1}{2\left(q-q^{-1}\right)^{2}}\left[2-e^{S(Z)-S\left(q^{2} Z\right)}-e^{S(Z)-S\left(q^{-2} Z\right)}\right]
$$

Notice that this Ricatti equation determines the value of $S$ at the point $q^{2} Z$ as long as the values of $S$ at the points $Z$ and $q^{-2} Z$ are known. In this sense, (A.9) should be considered as a second order finite difference equation. Besides, either (A.7), (A.8) and (A.9) become (A.1), (A.2) and (A.3), respectively, in the "classical" limit $q \longrightarrow 1$. Moreover, if $q$ were a $k$-th root of unity, then the $\theta$ variable could be restricted to take a discrete set of values, let us say $\theta=\frac{2 \pi n}{k}, \quad n=0,1, \cdots, k-1$, in which case we would obtain a $\mathbf{Z}_{k}$-invariant theory.

\section{B Exact FP-computation of $\mathrm{D}=1$ finite chains}

A suggestive feature of the FP-approach considered in this paper is that it provides a recipe to compute exactly the ground state and energy of a whole family of 1dimensional hamiltonians defined on a finite lattice, among which the ITF model of section 2 would constitute a particular case. Here we present the derivation of this result.

Firstly, let us consider the lattice configuration as a collection of 0's and 1's. The presence of a 1 (respec. a 0 ) at the site $j$ of the lattice should be thought of as equivalent to the existence (respec. absence) of a $\sigma^{z}$ at this position. Besides, let us assume that the number $N$ of sites of the lattice and the number of 1 's arranged on it are both even. Under these assumptions, it is not difficult to convince oneself that there exist just $2^{N-1}$ different possibilities to distribute a set of 0 's and 1's on the lattice. We can associate to each one of these possibilities a label $I_{a}$, namely:

$$
\left\{I_{a}\right\}_{a=1}^{2^{N-1}} \equiv\left\{I_{1}=(0,0, \stackrel{(N)}{\cdots}, 0), I_{2}=(1,1,0 \stackrel{(N)}{\cdots}, 0), \cdots, I_{2^{N-1}}=(1,1, \stackrel{(N)}{\cdots}, 1,1)\right\}
$$

Indeed, we can think in the labels $I_{a}$ as the elements of a certain equivalence class $\frac{\mathbf{Z}_{2}^{\otimes N}}{\mathcal{F}_{a}}$. Here $\mathbf{Z}_{2}$ reflects the sole presence of 0's and 1's in the lattice, with the usual rule of sum:

$$
1+0=1 \bmod 2 \quad, \quad 1+1=0 \bmod 2
$$

whereas $\mathcal{F}_{a}$ takes into account the fact that the number of 1 is constrained to be even:

$$
\mathcal{F}_{a}=\sum_{j=1}^{N} I_{a}(j)=0 \bmod 2
$$

Secondly, to each label $I_{a}$ we attach an operator $\sigma_{I_{a}}^{z}$ defined as follows: 


$$
\sigma_{I_{a}}^{z}=\prod_{j=1}^{N}\left(\sigma_{j}^{z}\right)^{I_{a}(j)}
$$

( to $I_{a}=(1,1,0,0,1,1)$ it would correspond the operator $\sigma_{I_{a}}^{z}=\sigma_{1}^{z} \sigma_{2}^{z} \sigma_{5}^{z} \sigma_{6}^{z}$, for example).

It is easy to see that the local operators $\sigma_{I_{a}}^{z}$ verify the fusion rule:

$$
\sigma_{I_{a}}^{z} \sigma_{I_{b}}^{z}=\sigma_{I_{a}+I_{b}}^{z}
$$

and, of course:

$$
\left(\sigma_{I_{a}}^{z}\right)^{2}=\mathbf{1}
$$

Likewise, the following commutation relation is satisfied:

$$
\sigma_{j}^{x} \sigma_{I_{a}}^{z}=(-1)^{I_{a}(j)} \sigma_{I_{a}}^{z} \sigma_{j}^{x}
$$

Finally, let us introduce a kind of generalized ITF hamiltonian, characterized by the fact that the interacting term includes couplings to all scales and to any number of spins:

$$
H=-\sum_{j=1}^{N} \sigma_{j}^{x}-\sum_{a=2}^{2^{N-1}} \lambda_{I_{a}} \sigma_{I_{a}}^{z}
$$

The question of finding the ground state and energy of the hamiltonian (B.6) can be faced as follows. Suppose we assume for the first the ansatz:

$$
\left|\psi_{0}\right\rangle=e^{-\frac{1}{2} S\left(\sigma_{1}^{z}, \cdots, \sigma_{N}^{z}\right)}|0\rangle
$$

but now with:

$$
S\left(\sigma_{1}^{z}, \cdots, \sigma_{N}^{z}\right)=-\sum_{a=2}^{2^{N-1}} \alpha_{I_{a}} \sigma_{I_{a}}^{z}
$$

and where the state $|0\rangle$ verify (3.3).

We want to obtain the FP hamiltonian associated to the ansatz (B.7), so we apply $H_{0}=-\sum_{j=1}^{N} \sigma_{j}^{x}$ on it, as to get:

$$
H^{F P}=-\sum_{j=1}^{N}\left(\sigma_{j}^{x}-e^{\Delta_{j}(S)}\right)=-\sum_{j=1}^{N}\left(\sigma_{j}^{x}-e^{-\sum_{a=2}^{2^{N-1}} I_{a}(j) \alpha_{I_{a}} \sigma_{I_{a}}^{z}}\right)
$$

A lengthy but straightforward calculation, where the use of eqs.(B.2)-(B.5) is essential, allows one to rewrite $(\mathbb{B . 9})$ as:

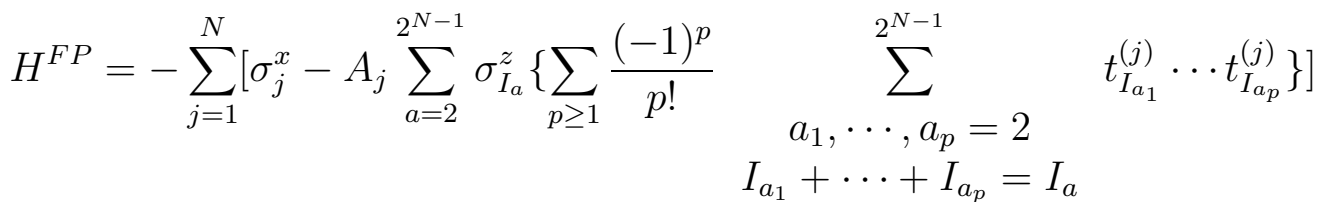

where we have introduced 


$$
\begin{gathered}
A_{j}=\prod_{a=2}^{2^{N-1}} \cosh \left[I_{a}(j) \alpha_{I_{a}}\right] \\
t_{I_{a}}^{(j)} \equiv I_{a}(j) t_{I_{a}} \equiv I_{a}(j) \tanh \alpha_{I_{a}}
\end{gathered}
$$

In fact, if we take translational invariance for granted, the value of $A_{j}$ remains invariable whatever be the site $j$ on the lattice; we may then express $A_{j} \equiv A, \forall j=$ $1, \cdots, N$. Assuming that this condition is fulfilled, the energy associated with (B.10) is given by:

$$
E^{F P}=-A\left[N+\sum_{j=1}^{N} \sum_{p \geq 1} \frac{(-1)^{p}}{p !} \sum_{\substack{a_{1}, \cdots, a_{p}=2 \\ I_{a_{1}}+\cdots+I_{a_{p}}=I_{1}}}^{2^{N-1}} t_{I_{a_{1}}}^{(j)} \cdots t_{I_{a_{p}}}^{(j)}\right]
$$

Moreover, the comparison between the $H^{F P}$ of $(\mathrm{B} .10)$ and the generic hamiltonian (B.6) suggests the identification:

$$
\lambda_{I_{a}}=-A \sum_{j=1}^{N} \sum_{p \geq 1} \frac{(-1)^{p}}{p !} \sum_{\substack{a_{1}, \cdots, a_{p}=2 \\ I_{a_{1}}+\cdots+I_{a_{p}}=I_{a}}}^{2^{N-1}} t_{I_{a_{1}}}^{(j)} \cdots t_{I_{a_{p}}}^{(j)} \quad, \quad a=2, \cdots, 2^{N-1}
$$

To make explicit these formulae, let us apply them to the ITF model of section 2 , but now for the case of a finite lattice with $N=4$ sites. In table 1 , we have summarized the notations and coupling constants appearing in this case, as well as the interaction terms these constants give account of.

A glance to table 1 suggests the choice

$$
\left|\psi_{0}\right\rangle=e^{\frac{1}{2} \alpha_{1} \sum_{j=1}^{4} \sigma_{j}^{z} \sigma_{j+1}^{z}+\frac{1}{4} \alpha_{2} \sum_{j=1}^{4} \sigma_{j}^{z} \sigma_{j+2}^{z}+\frac{1}{2} \alpha_{3} \sigma_{1}^{z} \sigma_{2}^{z} \sigma_{3}^{z} \sigma_{4}^{z}}|0\rangle
$$

where the factor $\frac{1}{4}$ in $(B .15)$ has been introduced in order to avoid overcounting in the corresponding term (for $N=4, \quad \sum_{j=1}^{4} \sigma_{j}^{z} \sigma_{j+1}^{z}=\sum_{j=1}^{4} \sigma_{j}^{z} \sigma_{j+3}^{z}$ ).

Now we require that $H_{I T F}^{F P}$ coincides with $H_{I T F}$ :

$$
H_{I T F}^{F P}=H_{I T F}=-\sum_{j=1}^{4} \sigma_{j}^{x}-\lambda \sum_{j=1}^{4} \sigma_{j}^{z} \sigma_{j+1}^{z}
$$

but since we have three different couplings it must be

$$
\begin{gathered}
t_{I_{2}}=t_{I_{3}}=t_{I_{4}}=t_{I_{5}} \equiv t_{1}=\tanh \alpha_{1} \\
t_{I_{6}}=t_{I_{7}} \equiv t_{2}=\tanh \alpha_{2} \\
t_{I_{8}} \equiv t_{3}=\tanh \alpha_{3}
\end{gathered}
$$


On the other hand, one is free to select a particular site, say $j=1$, to get:

$$
A=A_{1}=\prod_{a=2}^{8} \cosh \left[I_{a}(1) \alpha_{I_{a}}\right]=\frac{1}{\left(1-t_{1}^{2}\right)\left(1-t_{2}^{2}\right)^{1 / 2}\left(1-t_{3}^{2}\right)^{1 / 2}}
$$

Now, introducing eqs.(B.17)-(B.18) in (B.13)-( $\overline{B .14})$ we obtain:

$$
e_{I T F}^{F P}=-\frac{E_{I T F}^{F P}}{4}=\frac{1+t_{1}^{2} t_{2} t_{3}}{\left(1-t_{1}^{2}\right)\left(1-t_{2}^{2}\right)^{1 / 2}\left(1-t_{3}^{2}\right)^{1 / 2}}
$$

and

$$
\begin{gathered}
\lambda_{I_{2}}=\lambda_{I_{3}}=\lambda_{I_{4}}=\lambda_{I_{5}}=\lambda=\frac{2 t_{1}\left(1-t_{2}\right)\left(1-t_{3}\right)}{\left(1-t_{1}^{2}\right)\left(1-t_{2}^{2}\right)^{1 / 2}\left(1-t_{3}^{2}\right)^{1 / 2}} \\
\lambda_{I_{6}}=\lambda_{I_{7}}=0=\left(1-t_{3}\right)\left(t_{1}^{2}-t_{2}\right) \\
\lambda_{I_{8}}=0=t_{3}+t_{1}^{2} t_{2}
\end{gathered}
$$

Eqs.(B.19)-(B.20) can be easily solved, and in fact we obtain:

$$
e_{I T F}^{F P}=\frac{\left(1+t_{2}^{2}\right)^{1 / 2}}{1-t_{2}}
$$

where $t_{2}$ is the real and positive solution of the polinomial:

$$
t_{2}^{4}-\frac{4 t_{2}^{3}}{\lambda^{2}}-2 t_{2}^{2}-\frac{4 t_{2}}{\lambda^{2}}+1=0
$$

namely:

$$
t_{2}(\lambda)=\frac{1}{\lambda^{2}-1+\sqrt{1+\lambda^{4}}}\left[\lambda^{2}+1+\sqrt{1+\lambda^{4}}-2\left(\lambda^{2}+\sqrt{1+\lambda^{4}}\right)^{1 / 2}\right]
$$

Now if we substitute eq.(B.23) in (B.21) we find the final result:

$$
e_{I T F}^{F P}(\lambda)=\frac{1}{2}\left[\sqrt{1+\lambda^{2}+\lambda \sqrt{2}}+\sqrt{1+\lambda^{2}-\lambda \sqrt{2}}\right]
$$

This expression coincides precisely with the exact value given by the formula [3]:

$$
e_{I T F}(\lambda)=\frac{1}{N} \sum_{j=-\frac{N}{2}}^{\frac{N}{2}-1} \sqrt{1+\lambda^{2}+2 \lambda \cos \left[\frac{(2 j+1) \pi}{N}\right]}
$$

when we particularized to the lattice with $N=4$.

\section{Explicit computations in the FP-version of the ITF model}

In this appendix we deduce the formulae for the $t_{n}$ and $e_{N}^{F P}$ exhibited in section 4 .

The case $u_{N}=1$ is very easy to handle because the square roots appearing in eqs. (4.19)-(4.20) can be performed in terms of trigonometric functions. In particular, we get from eq. (4.15) 


$$
\hat{t}_{m}=-\sqrt{\frac{2 C_{N}}{N}} \sin \left(\frac{\pi m}{N}\right)
$$

and introducing this equation into eq.(4.17) we find:

$$
1=\frac{\sqrt{2 C_{N}}}{N} \sum_{m=0}^{N-1} \sin \left(\frac{\pi m}{N}\right)=\frac{\sqrt{2 C_{N}}}{N} \cot \left(\frac{\pi}{2 N}\right)
$$

From this euation the value for $C_{N}$ follows:

$$
C_{N}=\frac{1}{2}\left(N \tan \frac{\pi}{2 N}\right)^{2}
$$

On the other hand, working out eq. (4.19) with the help of (C.3) and (C.1) yields

$$
t_{n}(N)=\frac{\sin ^{2}\left(\frac{\pi}{2 N}\right)}{\sin \left[\frac{\pi}{N}\left(n+\frac{1}{2}\right)\right] \sin \left[\frac{\pi}{N}\left(n+\frac{1}{2}\right)\right]}
$$

In order to compute the energy $e_{N}^{F P}$ we rewrite eq.4.11) in the form

$$
e_{N}^{F P}=\prod_{n=1}^{N-1}\left(1-t_{n}\right)^{-\frac{1}{2}}\left(1+t_{n}\right)^{-\frac{1}{2}}
$$

Now we can make use of eq. C.4 to express the monomials of (C.5) as

$$
\begin{aligned}
& 1+t_{n}=\frac{\sin ^{2}\left(\frac{\pi n}{N}\right)}{\sin \left[\frac{\pi}{N}\left(n+\frac{1}{2}\right)\right] \sin \left[\frac{\pi}{N}\left(n+\frac{1}{2}\right)\right]} \\
& 1-t_{n}=\frac{\sin \left(\frac{\pi n}{N}+\frac{\theta_{N}}{2}\right) \sin \left(\frac{\pi n}{N}-\frac{\theta_{N}}{2}\right)}{\sin \left[\frac{\pi}{N}\left(n+\frac{1}{2}\right)\right] \sin \left[\frac{\pi}{N}\left(n+\frac{1}{2}\right)\right]}
\end{aligned}
$$

where $\theta_{N}$ was defined in equation (4.24).

Now using the formula

$$
\prod_{n=1}^{N-1} \sin \frac{\pi}{N}(n-a)=\frac{1}{2^{N-1}} \frac{\sin (\pi a)}{\sin \left(\frac{\pi a}{N}\right)}
$$

we obtain

$$
\begin{gathered}
\prod_{n=1}^{N-1}\left(1+t_{n}\right)=\left(N \sin \frac{\pi}{2 N}\right)^{2} \\
\prod_{n=1}^{N-1}\left(1-t_{n}\right)=\left(\frac{\sin \frac{\pi}{2 N} \sin \frac{N \theta_{N}}{2}}{\sin \frac{\theta_{N}}{2}}\right)^{2}
\end{gathered}
$$

From these equations and eq.(4.23) the eq. (C.3) follows directly.

Next, let us consider the case where $N \longrightarrow \infty$ and $0 \leq u_{\infty} \leq 1$. Now the sums can be converted into integrals, as for example eq.(4.19):

$$
t_{n}=-\int_{0}^{2 \pi} \frac{d x}{2 \pi} e^{i n x}\left(C_{\infty}-\frac{2 \lambda}{e_{\infty}^{F P}} \cos x\right)^{1 / 2}
$$




$$
=(-1)^{n+1} \frac{\sqrt{C_{\infty}}}{\pi} \int_{0}^{\pi} d x \cos (n x)\left(1+u_{\infty} \cos x\right)^{1 / 2}
$$

But replacing $\cos x=1-2 \sin ^{2}\left(\frac{x}{2}\right)$ in (C.11) and subsequently changing variables $\frac{x}{2} \longrightarrow x$ we get:

$$
t_{n}=(-1)^{n+1} \frac{2}{\pi} \sqrt{C_{\infty}\left(1+u_{\infty}\right)} \int_{0}^{\frac{\pi}{2}} \cos (2 n x)\left(1-\frac{2 u_{\infty}}{1+u_{\infty}} \sin ^{2} x\right)^{1 / 2}
$$

This integral can be performed if we first expand the square root according to the formula:

$$
(1-Z)^{\alpha}=\sum_{m=0}^{\infty} \frac{\Gamma(m-\alpha)}{\Gamma(-\alpha) \Gamma(m+1)} Z^{m}
$$

and then we use the result

$$
\int_{0}^{\frac{\pi}{2}} d x \cos (2 n x) \sin ^{2 m} x= \begin{cases}\frac{(-1)^{n}}{2^{2 m}} \frac{\Gamma(2 m+1)}{\Gamma(m+n+1) \Gamma(m-n+1)} & m \geq n \\ 0 & m<n\end{cases}
$$

Finally, we obtain the expression

$$
t_{n}=\frac{\sqrt{2 C_{\infty}}}{8 \pi} \frac{u_{\infty}^{n}}{\left(1+u_{\infty}\right)^{n-\frac{1}{2}}} \frac{\Gamma\left(\frac{n}{2}-\frac{1}{4}\right) \Gamma\left(\frac{n}{2}+\frac{1}{4}\right)}{\Gamma(n+1)} F\left(n-\frac{1}{2}, n+\frac{1}{2}, 2 n+1 ; \frac{2 u_{\infty}}{1+u_{\infty}}\right)
$$

The equation which yields $C_{\infty}$ is obtained by setting $t_{0}=-1$ in eq.(C.15), namely

$$
\sqrt{C_{\infty}}\left(1+u_{\infty}\right)^{1 / 2} F\left(-\frac{1}{2}, \frac{1}{2}, 1 ; \frac{2 u_{\infty}}{1+u_{\infty}}\right)=1
$$

Taking the square of eq.(C.16) and using the fact that $C_{\infty}=\frac{2 \lambda}{e_{\infty}^{F P} u_{\infty}}$, we deduce an equation for $e_{\infty}^{F P}$ as a function of $\lambda$ and $u_{\infty}$ :

$$
e_{\infty}^{F P}=2 \lambda\left(\frac{1+u_{\infty}}{u_{\infty}}\right) F^{2}\left(-\frac{1}{2}, \frac{1}{2}, 1 ; \frac{2 u_{\infty}}{1+u_{\infty}}\right)
$$

Replacing now $u_{\infty}$ in terms of the parameter $\lambda_{R}$ introduced in (4.35) one obtains eq. (4.34).

As for the equation (4.33) for the $t_{n}$ 's, it is easily derived from (4.35) and (C.15)(C.16) . Likewise, expression (4.34) is obtained by taking the $N \longrightarrow \infty$ limit of (C.5):

$$
e_{\infty}^{F P}=\prod_{n=1}^{\infty}\left(1-t_{n}^{2}\right)^{-1}
$$

and using (C.15). Notice that the exponent in eq. (C.18) is -1 and not $-\frac{1}{2}$ as in eq. (C.5). This takes care of the constraint $t_{n}=t_{N-n}$ which in the $N \longrightarrow \infty$ limit becomes $t_{n}=t_{-n}$, with $n \in \mathbf{Z}$. 
Eq. (C.15) for $u_{\infty}=1$ gives the value $t_{n}=\frac{1}{4 n^{2}-1}$. If $u_{\infty}<1$ we can also find an indication of the behaviour of the $t_{n}$, just on condition that $n$ be large enough. To this end, we can carry out a saddle point evaluation for $t_{n}$ on the basis of the standard integral representation of hypergeometric functions [24]. Indeed, we can write (C.15) as:

$$
t_{n}=\frac{\sqrt{C_{\infty}\left(1+u_{\infty}\right)}}{2 \pi} \frac{z^{n}}{\left(n-\frac{1}{2}\right)} \int_{0}^{1} d t\left[\frac{t(1-t)}{1-z t}\right]^{n-\frac{1}{2}}
$$

where we have introduced the parameter

$$
z=\frac{2 u_{\infty}}{1+u_{\infty}}
$$

Due to the particularly compact form of the integrand, the saddle point in eq. (C.19) turns out to be independent of $n$. Our result is

$$
\begin{aligned}
& t_{n} \simeq \frac{1}{2} \sqrt{\frac{C_{\infty}}{\pi}} \frac{1}{\left(n-\frac{1}{2}\right)^{\frac{3}{2}}} \\
& \times\left(\frac{1+\sqrt{1-u_{\infty}^{2}}}{1+u_{\infty}+\frac{1}{2} \sqrt{1-u_{\infty}^{2}}}\right)^{\frac{1}{2}}\left(\frac{1-u_{\infty}}{1+u_{\infty}}\right)^{\frac{1}{4}}\left(\frac{1+\sqrt{1-u_{\infty}^{2}}}{u_{\infty}}\right)^{-n} n>>1
\end{aligned}
$$

Finally, the expression (4.35) can be used to replace $u_{\infty}$ by $\lambda_{R}$ and in this way we get eq.(4.39).

\section{Higher dimensional ansatzs}

One of the reasons to justify the introduction of new ansatzs for the ground state of the $1 D$ ITF model, amounts to our wish of discussing higher dimensional models for which exact solutions are not known. In this sense, the obvious generalization of the $1 D$-dimensional ansatz proposed in eq.(4.4) becomes

$$
\left|\psi\left(\left\{\alpha_{\mathbf{r}}\right\}\right)\right\rangle=\exp \left(\frac{1}{4} \sum_{\mathbf{r} \in \Lambda^{\prime}} \sum_{\mathbf{j} \in \Lambda} \alpha_{\mathbf{r}} \sigma_{\mathbf{j}}^{z} \sigma_{\mathbf{j}+\mathbf{r}}^{z}\right)|0\rangle
$$

where $\Lambda$ is the hypercubic lattice $\mathbf{Z}_{L}^{D}$ and $\Lambda^{\prime}=\Lambda-\{0\}$. We shall suppose that $\alpha_{\mathbf{r}}=\alpha_{-\mathbf{r}}$ with $\mathbf{r} \in \Lambda^{\prime}$.

The FP-Hamiltonian corresponding to (D.1) has the form

$$
H^{F P}=-\sum_{\mathbf{j} \in \Lambda}\left[\sigma_{\mathbf{j}}^{x}-e^{-\sum_{\mathbf{r} \in \Lambda^{\prime}} \alpha_{\mathbf{r}} \sigma_{\mathbf{j}}^{z} \sigma_{\mathbf{j}+\mathbf{r}}^{z}}\right]
$$

Expanding eq.(D.2) we arrive to a generalized ITF model

$$
H^{F P}=-E^{F P}-\sum_{\mathbf{j} \in \Lambda} \sigma_{\mathbf{j}}^{x}-\frac{1}{2} \sum_{\mathbf{j} \in \Lambda} \lambda_{\mathbf{r}} \sigma_{\mathbf{j}}^{z} \sigma_{\mathbf{j}+\mathbf{r}}^{z}+\left(4 \sigma^{z \prime} \mathbf{s}\right)+\cdots
$$

with 


$$
\begin{gathered}
E^{F P}=-N e_{N}^{F P}=-N \prod_{\mathbf{r} \in \Lambda^{\prime}} \cosh \alpha_{\mathbf{r}} \\
\frac{\lambda_{\mathbf{r}}}{e_{N}^{F P}}=-\sum_{\mathbf{n} \in \Lambda} t_{\mathbf{n}} t_{\mathbf{r}-\mathbf{n}}
\end{gathered}
$$

and

$$
\begin{gathered}
t_{\mathbf{0}}=-1 \\
t_{\mathbf{r}}=\tanh \alpha_{\mathbf{r}}, \quad \mathbf{r} \neq \mathbf{0}
\end{gathered}
$$

To solve eqs.(D.4)-(D.5) we introduce the Fourier transformation

$$
t_{\mathbf{n}}=\frac{1}{L^{D / 2}} \sum_{\mathbf{m} \in \Lambda^{*}} e^{2 \pi i \frac{\mathrm{nm}}{L}} \hat{t}_{\mathbf{m}}
$$

where $\Lambda^{*}$ is the dual lattice of $\Lambda$. For a hypercubic lattice both $\Lambda^{*}$ and $\Lambda$ coincide. Following the same steps of section 4 , we find

$$
\hat{t}_{\mathbf{m}}=-\frac{\varepsilon_{\mathbf{m}}}{L^{D / 2}}\left(C_{N}-\sum_{\mathbf{r} \in \Lambda^{\prime}} e^{-2 \pi i \frac{\mathrm{rm}}{L}} \frac{\lambda_{\mathbf{r}}}{e_{N}^{F P}}\right)^{1 / 2}
$$

where

$$
C_{N}=\sum_{\mathbf{m} \in \Lambda^{*}} \hat{t}_{\mathbf{m}}^{2}=\sum_{\mathbf{n} \in \Lambda} t_{\mathbf{n}}^{2}
$$

and $\varepsilon_{\mathbf{m}}=\varepsilon_{-\mathbf{m}}= \pm 1$. For convenience, we shall fix $\varepsilon_{\mathbf{m}}=1$.

In the limit $N \longrightarrow$ the $t_{\mathbf{n}}$ are given by

$$
t_{\mathbf{n}}=-\int_{0}^{2 \pi} \frac{d k_{1}}{2 \pi} \cdots \int_{0}^{2 \pi} \frac{d k_{D}}{2 \pi} e^{i \mathbf{n k}}\left[C_{\infty}-\frac{2}{e_{\infty}^{F P}} \sum_{a=1}^{D} \lambda_{a} \cos k_{a}\right]^{1 / 2}
$$

where $\lambda_{a}$ is the value of the coupling constant multiplying the operator $\sigma_{\mathbf{j}}^{z} \sigma_{\mathbf{j}+\mu_{a}}^{z}$ in eq.(D.3) ( $\mu_{a}$ is the unit vector along the $a^{\text {th }}$-direction).

In the special case of $D=2$ the integrals of (D.10) can be explicitly determined in a way which goes parallel to the followed in appendix C. Writting $\mathbf{n}=\left(n_{1}, n_{2}\right)$ and making use of eq.(D.6) we obtain after a lengthy computation

$$
\begin{gathered}
e_{\infty}^{F P}=\frac{2\left(\lambda_{1}+\lambda_{2}\right)}{\pi^{5}\left(\lambda_{R_{1}}+\lambda_{R_{2}}\right)} \\
\times\left[\left(1+\lambda_{R_{1}}+\lambda_{R_{2}}\right) F_{2}\left(-\frac{1}{2}, \frac{1}{2}, \frac{1}{2}, 1,1, \frac{4 \lambda_{R_{1}}}{\left(1+\lambda_{R_{1}}+\lambda_{R_{2}}\right)^{2}}, \frac{4 \lambda_{R_{2}}}{\left(1+\lambda_{R_{1}}+\lambda_{R_{2}}\right)^{2}}\right)\right]^{2}
\end{gathered}
$$

and 


$$
\begin{gathered}
t_{n_{1}, n_{2}}=\frac{1}{2 \pi \sqrt{\pi}} \\
\times\left[\frac{4 \lambda_{R_{1}}}{\left(1+\lambda_{R_{1}}+\lambda_{R_{2}}\right)^{2}}\right]^{n_{1}}\left[\frac{4 \lambda_{R_{2}}}{\left(1+\lambda_{R_{1}}+\lambda_{R_{2}}\right)^{2}}\right]^{n_{2}} \frac{\Gamma\left(n_{1}+n_{2}-\frac{1}{2}\right) \Gamma\left(n_{1}+\frac{1}{2}\right) \Gamma\left(n_{2}+\frac{1}{2}\right)}{\Gamma\left(2 n_{1}+1\right) \Gamma\left(2 n_{2}+1\right)} \\
\times \frac{F_{2}\left(n_{1}+n_{2}-\frac{1}{2}, n_{1}+\frac{1}{2}, n_{2}+\frac{1}{2}, 2 n_{1}+1,2 n_{2}+1, \frac{4 \lambda_{R_{1}}}{\left(1+\lambda_{R_{1}}+\lambda_{R_{2}}\right)^{2}}, \frac{4 \lambda_{R_{2}}}{\left(1+\lambda_{R_{1}}+\lambda_{R_{2}}\right)^{2}}\right)}{F_{2}\left(-\frac{1}{2}, \frac{1}{2}, \frac{1}{2}, 1,1, \frac{4 \lambda_{R_{1}}}{\left(1+\lambda_{R_{1}}+\lambda_{R_{2}}\right)^{2}}, \frac{4 \lambda_{R_{2}}}{\left(1+\lambda_{R_{1}}+\lambda_{R_{2}}\right)^{2}}\right)}
\end{gathered}
$$

where by $F_{2}$ we denote one of the hypergeometric series in two variables [24]. The parameters $\lambda_{R_{a}}, a=1,2$ play the role of "dressed" coupling constants and are defined through

$$
\frac{\lambda_{a}}{C_{\infty} e_{\infty}^{F P}}=\frac{\lambda_{R_{a}}}{1+\left(\lambda_{R_{1}}+\lambda_{R_{2}}\right)^{2}} \quad, \quad a=1,2
$$

From these formulae it is possible to investigate the existence of critical behaviour in the same way as we did in section 4 for the one dimensional case. Nevertheless, this analysis can be carried out directly from eq.(D.10). To this respect, we impose the condition

$$
C_{\infty}=\frac{2}{e_{\infty}^{F P}} \sum_{a=1}^{D} \lambda_{a}
$$

For large values of $|\mathbf{n}|$ the integral (D.10) is dominated by the small momenta $|\mathbf{k}|<\epsilon$, where $\epsilon \sim \frac{1}{|\mathbf{n}|}$ is a cutoff; namely

$$
t_{\mathbf{n}} \simeq-\frac{1}{e_{\infty}^{F P}} \int_{|\mathbf{k}|<\epsilon} \prod_{a=1}^{D} \frac{d k_{a}}{2 \pi} e^{i \mathbf{n k}}\left(\sum_{a=1}^{D} \lambda_{a} k_{a}^{2}\right)^{1 / 2}
$$

and assuming isotropy, i.e $\lambda_{a}=\lambda, \forall a$, we obtain the scaling behaviour

$$
t_{\mathbf{n}} \sim \frac{1}{|\mathbf{n}|^{D+1}}
$$

Away from the condition (D.14) we shall define a "mass parameter" $\mu^{2}$ as

$$
\mu^{2}=\frac{C_{\infty} e_{\infty}^{F P}}{\lambda}-2 D
$$

Because the isotropy, the relation between $\mu$ and the sole "dressed" coupling constant $\lambda_{R}$ existing in this case, comes from (D.13) and is given by

$$
\mu=\frac{1-D \lambda_{R}}{\sqrt{\lambda_{R}}}
$$

In terms of $\mu$, the behaviour of the $t_{\mathbf{n}}$ for large values of $|\mathbf{n}|$ is dictated by

$$
t_{\mathbf{n}} \simeq-\sqrt{\frac{\lambda}{e_{\infty}^{F P}}} \int_{|\mathbf{k}|<\epsilon} \prod_{a=1}^{D} \frac{d k_{a}}{2 \pi} e^{i \mathbf{n k}}\left(\mu^{2}+|\mathbf{k}|^{2}\right)^{1 / 2}
$$


Moreover, a saddle point evaluation of this integral for $\mu^{2}>0$ yields the exponential decaying law

$$
t_{\mathbf{n}} \sim \frac{1}{|\mathbf{n}|^{\frac{D}{2}+1}} e^{-\mu|\mathbf{n}|}
$$

Hence, $1 / \mu$ defines the correlation length.

The constraint $t_{\mathbf{0}}=-1$ gives us an expresion where the density of energy $e_{\infty}^{F P}$ and the parameter $\mu$ are tied together, namely

$$
\left(\frac{e_{\infty}^{F P}}{\lambda}\right)^{1 / 2}=\int_{0}^{2 \pi} \frac{d k_{1}}{2 \pi} \cdots \int_{0}^{2 \pi} \frac{d k_{D}}{2 \pi}\left[\mu^{2}+4 \sum_{a=1}^{D} \sin ^{2}\left(\frac{k_{a}}{2}\right)\right]^{1 / 2}
$$

To investigate the singular behaviour of $e_{\infty}^{F P}$ as a function of $\mu$, it is sufficient to consider the small values of the "momenta" $k_{a}$ as we did previously for the $t_{\mathbf{n}}$. In this case the integral (D.21) becomes proportional to the function $F_{D}(\mu, \epsilon)$ defined as

$$
F_{D}(\mu, \epsilon)=\int_{0}^{\epsilon} d k k^{D-1}\left(\mu^{2}+k^{2}\right)^{1 / 2}
$$

where $\epsilon$ is a cutoff. Studying the singular behaviour of $F_{D}(\mu, \epsilon)$ in the vecinity of the point $\mu=0$ we get:

$$
F_{D}(\mu, \epsilon)= \begin{cases}c_{D} \mu^{D+1} \log \left(\frac{\mu}{\epsilon}\right)+F_{D}^{r e g}(\mu, \epsilon) & \text { for } D \text { odd } \\ F_{D}^{r e g}(\mu, \epsilon) & \text { for } D \text { even }\end{cases}
$$

where $F_{D}^{r e g}(\mu, \epsilon)$ denotes a regular function at $\mu=0$. Eq. (D.23) means that only in odd dimensions $(D=3,5, \cdots)$ we obtain a singular logarithmic behaviour; that is somehow the generalization of the one dimensional result (4.38). The only difference with this latter is the power $\mu^{D+1}$ in front of the logarithm, which is expected from dimensional grounds.

Curiously enough, we do not observe singularities in the ground state energy if the dimension is even. This fact does not exclude however the existence of discontinuities in some derivative of the energy as we cross the critical point, just as it occurs in a mean field calculation.

In any case, it is clear that the ansatz (D.1) does not lead for $D>1$ to the standard ITF model. That supposes, according to the general ideas expressed in the introduction of the paper, that we have to return to perturbation theory and figure out the terms necessary to improve the ansatz. In $D=2$ for example, we have neglected in the ansatz the terms involving four $\sigma^{z}$ matrices. We claim here that these terms are important if one wants to get closer to the ITF model. As a result, we propose the following ansatz for $D \geq 2$

$$
\left|\psi\left(\left\{\alpha_{\mathbf{r}_{1}, \mathbf{r}_{2}, \mathbf{r}_{3}}\right\}\right)\right\rangle=\exp \left(\sum_{\mathbf{r}_{\mathbf{s}} \in \Lambda^{\prime}} \frac{\alpha_{\mathbf{r}_{1}, \mathbf{r}_{2}, \mathbf{r}_{3}}}{2 n_{\mathbf{r}_{1}, \mathbf{r}_{2}, \mathbf{r}_{3}}} \sum_{\mathbf{j} \in \Lambda} \sigma_{\mathbf{j}}^{z} \sigma_{\mathbf{j}+\mathbf{r}_{1}}^{z} \sigma_{\mathbf{j}+\mathbf{r}_{2}}^{z} \sigma_{\mathbf{j}+\mathbf{r}_{3}}^{z}\right)|0\rangle
$$

where $n_{\mathbf{r}_{1}, \mathbf{r}_{2}, \mathbf{r}_{3}}$ is defined by 


$$
n_{\mathbf{r}_{1}, \mathbf{r}_{2}, \mathbf{r}_{3}}= \begin{cases}2 & \text { if } \mathbf{r}_{a}=\mathbf{r}_{b} \neq \mathbf{r}_{c} \\ 4 & \text { otherwise }\end{cases}
$$

Equation (D.24) includes the ansatz (D.1) by allowing two $\mathbf{r}^{\prime}$ s to coincide. The quantities (D.25) accounts for the overcounting in the sum over $\mathbf{j}$ in the exponent of (D.24). Likewise the parameters $\alpha_{\mathbf{r}_{1}, \mathbf{r}_{2}, \mathbf{r}_{3}}$ has to be totally symmetric in their three indices and must satisfy the reflection symmetry property

$$
\alpha_{\mathbf{r}_{1}, \mathbf{r}_{2}, \mathbf{r}_{3}}=\alpha_{-\mathbf{r}_{1}, \mathbf{r}_{2}-\mathbf{r}_{1}, \mathbf{r}_{3}-\mathbf{r}_{1}}=\alpha_{\mathbf{r}_{1}-\mathbf{r}_{2},-\mathbf{r}_{2}, \mathbf{r}_{3}-\mathbf{r}_{2}}=\alpha_{\mathbf{r}_{1}-\mathbf{r}_{3}, \mathbf{r}_{2}-\mathbf{r}_{3},-\mathbf{r}_{3}}
$$

which is the analogue of condition $\alpha_{r}=\alpha_{-r}$ in the one dimensional case.

The FP-Hamiltonian associated to (D.24) is

$$
H^{F P}=-\sum_{\mathbf{j} \in \Lambda}\left[\sigma_{\mathbf{j}}^{x}-\exp \left(-\sum_{\mathbf{r}^{\prime} \mathbf{s} \in \Lambda^{\prime}} \alpha_{\mathbf{r}_{1}, \mathbf{r}_{2}, \mathbf{r}_{3}} \sigma_{\mathbf{j}}^{z} \sigma_{\mathbf{j}+\mathbf{r}_{1}}^{z} \sigma_{\mathbf{j}+\mathbf{r}_{2}}^{z} \sigma_{\mathbf{j}+\mathbf{r}_{3}}^{z}\right)\right]
$$

and can be expanded as

$$
H^{F P}=-E^{F P}-\sum_{\mathbf{j} \in \Lambda} \sigma_{\mathbf{j}}^{x}-\sum_{\mathbf{r}^{\prime} \mathbf{s} \in \Lambda^{\prime}} \frac{\lambda_{\mathbf{r}_{1}, \mathbf{r}_{2}, \mathbf{r}_{3}}}{\left(n_{\mathbf{r}_{1}, \mathbf{r}_{2}, \mathbf{r}_{3}}\right) !} \sum_{\mathbf{j} \in \Lambda} \sigma_{\mathbf{j}}^{z} \sigma_{\mathbf{j}+\mathbf{r}_{1}}^{z} \sigma_{\mathbf{j}+\mathbf{r}_{2}}^{z} \sigma_{\mathbf{j}+\mathbf{r}_{3}}^{z}+\left(6 \sigma^{z \prime} \mathbf{s}\right)+\cdots
$$

Compairing (D.27) and (D.28) and using the same trick of section 4, according to which one could replace consistently the $\sigma_{\mathbf{j}}^{z}$ by $\sigma_{\mathbf{j}}= \pm 1$, we obtain the following equations for $E^{F P}$ and $\lambda_{\mathbf{r}_{1}, \mathbf{r}_{2}, \mathbf{r}_{3}}$ as functions of $\alpha_{\mathbf{r}_{1}, \mathbf{r}_{2}, \mathbf{r}_{3}}$

$$
\begin{gathered}
E^{F P}=-\frac{N}{2^{N}} \sum_{\sigma^{\prime} \mathbf{s}} \exp \left(-\sum_{\mathbf{r}^{\prime} \mathbf{s} \in \Lambda^{\prime}} \alpha_{\mathbf{r}_{1}, \mathbf{r}_{2}, \mathbf{r}_{3}} \sigma_{\mathbf{0}} \sigma_{\mathbf{r}_{1}} \sigma_{\mathbf{r}_{2}} \sigma_{\mathbf{r}_{3}}\right) \\
\lambda_{\mathbf{s}_{1}, \mathbf{s}_{2}, \mathbf{s}_{3}}=-\frac{1}{2^{N}} \sum_{\sigma^{\prime} \mathbf{s}}\left(\sum_{\mathbf{j} \in \Lambda} \sigma_{\mathbf{j}} \sigma_{\mathbf{j}+\mathbf{s}_{1}} \sigma_{\mathbf{j}+\mathbf{s}_{2}} \sigma_{\mathbf{j}+\mathbf{s}_{3}}\right) \exp \left(-\sum_{\mathbf{r}^{\prime} \mathbf{s} \in \Lambda^{\prime}} \alpha_{\mathbf{r}_{1}, \mathbf{r}_{2}, \mathbf{r}_{3}} \sigma_{\mathbf{0}} \sigma_{\mathbf{r}_{1}} \sigma_{+\mathbf{r}_{2}} \sigma_{\mathbf{r}_{3}}\right)
\end{gathered}
$$

Naturally the problem which arises now is to perform the sum over the $\sigma$ 's. Observe that eq.(D.29) is a kind of classical partition function of a lattice involving couplings between 2 and 4 spins; in some sense, it recalls an 8-vertex model [1] so that there are chances to perform the sum, at least in some particular cases.

Finally, there is the question of inverting eq.(D.30) in order to express the $\alpha$ 's as functions of the $\lambda$ 's. Notice that the $\lambda$ 's could be thought of as a kind of correlators among $\sigma$ 's for a partition function as the one of eq.(D.29).

In summary, the problem of constructing the ground state for the $2 D$ quantum ITF model is essentially reduced, by means of the ansatz (D.24), to that of computing a $2 D$ nonlocal partition function followed by the inversion of the relation between certain correlators and coupling constants. The difficulties of these two tasks are considerable but we expect that the FP procedure can throw some light on the original unsolved problem. 


\section{References}

[1] R.J. Baxter, "Exactly Solved Models in Statistical Mechanics", Academic London (1982).

[2] T.D. Schultz, D.C. Mattis and E.H. Lieb, Rev. Mod. Phy 36, 856 (1964).

[3] P.Pfeuty, Ann.Phys.N.Y.57, 570 (1969).

[4] D.P Arovas and S.M Girvin, "Exact questions to some interesting answers in many body physics". Preprint 1991. Unpublished.

[5] G.D. Mahan, "Many Particle Physics", second edition. Plenum, New York, (1990) and references therein.

[6] R.Jastrow, Phys.Rev98,1479 (1955).

[7] Ian. Affleck, T.Kennedy, E.H. Lieb and H. Tasaki, Phys. Rev. Lett 59 , 799 (1987); Comm. Math. Phys 115, 477 (1988).

[8] R.B Laughlin, Phys. Rev. 114A, 331 (1986).

[9] J.G. Esteve and G. Sierra, Phys. Rev. B to be published.

[10] G. Parisi, "Statistical Field Theory", Addison-Wesley (1988).

[11] G. Parisi and Wu Yong-Shi, Sci.Sin 24, 483 (1981).

[12] J.Greensite and M.B. Halpern, Nucl.Phys. B242 , 167 (1984).

[13] J.Greensite, Nucl.Phys. B361, 729 (1991), Nucl.Phys. B390 , 439 (1993).

[14] E.Marinari, G. Parisi, Phys.Lett. B240, 375 (1990).

[15] J. Miramontes, J. Guillén, M.A. Vozmediano, Phys.Lett. B253, 38 (1991).

[16] E. Witten, Nucl.Phys. B188, 513 (1981).

[17] A. V. Turbiner, Usp. Fiz. Nank 144,35 (1984) [Sov. Phys.Usp 27,668 (1984)] and references therein.

[18] J.Greensite, Nucl.Phys. B158, 469 (1979).

[19] V. G. Drinfeld, "Quantum Groups" in Proceedings of the 1986 International Congress of Mathematics, ed. A. M. Gleason. AMS, Berkeley, (1987).

[20] M. Jimbo, "Quantum Groups and the Yang-Baxter Equation". Springer, Tokyo, (1991).

[21] P.G. Gennes, Solid. State Comm.1, 443 (1963).

[22] P. Pfeuty, Thesis, Paris (1970).

[23] R.B. Stinchcombe, J. Phys C6, 2459 (1973). 
[24] A. Erdelyi, W. Magnus, F. Oberhettinger, F.G. Tricomi, Higher Trascendental Functions, McGraw-Hill, Vol I, (1953).

[25] F. Jiménez, G. Sierra. Work in progress.

[26] E. Fradkin, S. Rabi, Phys. Rev.20D , 2566 (1979).

[27] M.A. Martín-Delgado, G. Sierra, "Strongly interacting spinless fermions in D=1 and 2 dimensions: A perturbative-variational approach". Preprint 1995. 


\section{Table captions}

Table1: Values of the coupling constants and interaction terms in the case of a ITF model defined on a finite lattice with $N=4$ sites. 


\section{Figure captions}

Figure 1a).- The shadow region simbolizes the possible non vanishing entries of the matrix $p_{n, I}$.

Figure 1b).- Information obtained up to order $\nu$ in perturbation theory.

Figure 1c).- Information obtained up to order $\nu$ using a truncated cluster expansion.

Figure 1d).- Here we choose one or more representants for each order in perturbation theory and construct a Hamiltonian which belongs to the same universality class of the original Hamiltonian.

Figure 2).- Comparison of the values for minus the 1D energy density in the cases exact $e_{I s i n g, D=1}$ (strong solid line), in the first order FP-aproximation $e_{I s i n g, D=1}^{F P,(\text { solid }}$ line) and in the second order FP-aproximation $e_{I s i n g, D=1}^{F P,(2)}$ (grey line).

Figure 3).- Action of the operator $\Delta$ on a generic state $\left|I_{a}\right\rangle=\left|\mu_{1}, \cdots, \mu_{N}\right\rangle$. 
Table 1

\begin{tabular}{|l|c|c|c|}
\hline \multicolumn{1}{|c|}{ Labels } & Interaction & $\begin{array}{c}\text { Coupling constant } \\
\text { in the ansatz }\end{array}$ & $\begin{array}{c}\text { Coupling constant } \\
\text { in the hamiltonian } H^{F P}\end{array}$ \\
\hline \hline$I_{1}=(0,0,0,0)$ & $\emptyset$ & $\emptyset$ & $\emptyset$ \\
$I_{2}=(1,1,0,0)$ & $\sigma_{j}^{z} \sigma_{j+1}^{z}$ & & \\
$I_{3}=(0,1,1,0)$ & & $\alpha_{1}$ & \\
$I_{4}=(0,0,1,1)$ & & & \\
$I_{5}=(1,0,0,1)$ & & $\alpha_{2}$ & 0 \\
\hline$I_{6}=(1,0,1,0)$ & $\sigma_{j}^{z} \sigma_{j+2}^{z}$ & & \\
$I_{7}=(0,1,0,1)$ & & & \\
\hline \hline
\end{tabular}

